\title{
Hippocampal Sequences During Exploration: Mechanisms and Functions
}

\author{
Céline Drieu ${ }^{* t}$ and Michaël Zugaro* \\ Center for Interdisciplinary Research in Biology, Collège de France, CNRS UMR 7241, INSERM U 1050, PSL Research \\ University, Paris, France
}

OPEN ACCESS

Edited by:

Francesca Cacucci, University College London, United Kingdom

Reviewed by:

Romain Goutagny, UMR7364 Laboratoire de Neurosciences Cognitives et Adaptatives (LNCA), France Caswell Barry, University College London, United Kingdom

*Correspondence:

Céline Drieu cdrieu1@jhu.edu Michaël Zugaro michael.zugaro@college-de-france.fr

${ }^{\dagger}$ Present Address: Céline Drieu,

Department of Psychological and Brain Sciences, Krieger School of Arts and Sciences, Johns Hopkins University, Baltimore, $M D$, United States

Specialty section:

This article was submitted to

Cellular Neurophysiology,

a section of the journal

Frontiers in Cellular Neuroscience

Received: 09 November 2018

Accepted: 08 May 2019

Published: 13 June 2019

Citation:

Drieu C and Zugaro M (2019)

Hippocampal Sequences During

Exploration: Mechanisms and

Functions.

Front. Cell. Neurosci. 13:232. doi: 10.3389/fncel.2019.00232
Although the hippocampus plays a critical role in spatial and episodic memories, the mechanisms underlying memory formation, stabilization, and recall for adaptive behavior remain relatively unknown. During exploration, within single cycles of the ongoing theta rhythm that dominates hippocampal local field potentials, place cells form precisely ordered sequences of activity. These neural sequences result from the integration of both external inputs conveying sensory-motor information, and intrinsic network dynamics possibly related to memory processes. Their endogenous replay during subsequent sleep is critical for memory consolidation. The present review discusses possible mechanisms and functions of hippocampal theta sequences during exploration. We present several lines of evidence suggesting that these neural sequences play a key role in information processing and support the formation of initial memory traces, and discuss potential functional distinctions between neural sequences emerging during theta vs. awake sharp-wave ripples.

\section{Keywords: place cell, network dynamics, phase precession, theta sequences, awake replay, memory}

\section{INTRODUCTION}

The ability of brain circuits to generate precise sequences of neuronal activity may underlie certain forms of sensory-motor processing and learning (Skaggs et al., 1996; Riehle et al., 1997; Stopfer et al., 1997; Petersen et al., 2001). While in some systems these sequences may be driven by external stimuli or motor signals, reports of internally generated neuronal sequences in the hippocampus, under either minimal (e.g., sleep; Lee and Wilson, 2002; Ji and Wilson, 2007) or invariant (Pastalkova et al., 2008; Wang et al., 2015) sensory-motor inputs, have identified a potential evolutionary mechanism for planning and memory consolidation (Lisman and Redish, 2009; Foster and Knierim, 2012; Buzsáki and Moser, 2013; Wikenheiser and Redish, 2015a; Zielinski et al., 2017).

The hippocampal network receives highly integrated inputs from distributed areas (Witter et al., 1989; Andersen et al., 2007; van Strien et al., 2009) and is endowed with auto-associative properties (Gilbert and Brushfield, 2009), which allows it to integrate and retrieve spatial as well as non-spatial information (Buzsáki and Moser, 2013; Eichenbaum, 2017). Bilateral lesions of the hippocampus result in long term deficits in episodic memory (Scoville and Milner, 1957) as well as in strong impairments in spatial memory (Morris et al., 1982). Indeed, the hippocampal formation has been proposed to support a cognitive map of the environment (Tolman, 1948; O'Keefe and Nadel, 1978), based on the remarkable property of its "place cells" that discharge when the animal occupies restricted locations in the environment ("place fields," O'Keefe and Dostrovsky, 1971). During the exploration, the animal sequentially crosses successive place fields, and the corresponding 
place cells fire in sequence, reflecting the ongoing trajectory at the behavioral timescale. Strikingly, embedded in these slow sequences, time-compressed sequences emerge in each cycle of the ongoing theta rhythm (O'Keefe and Recce, 1993; Skaggs et al., 1996), a strong and regular $\sim 8 \mathrm{~Hz}$ oscillation of the hippocampal local field potential (LFP; Jouvet, 1969; Vanderwolf, 1969). These sequences are known as "theta sequences." As a result of this fast timescale organization, the spikes emitted by successive cell assemblies occur within dozens of milliseconds, and this pattern is continually repeated in successive theta cycles, which is conducive to Hebbian synaptic plasticity (Skaggs et al., 1996; Magee and Johnston, 1997; Stuart, 2001; Buzsáki and Draguhn, 2004; Wójtowicz and Mozrzymas, 2015). Therefore, theta sequences have been proposed to allow the formation of spatial and episodic memory traces, by anchoring spatial events in their temporal context (Skaggs et al., 1996; Drieu et al., 2018).

Interestingly, fast time-compressed trajectories are also represented by neuronal sequences that occur during brief pauses and prolonged immobility (Foster and Wilson, 2006; Diba and Buzsáki, 2007), when theta oscillations are absent. Instead, these replay events occur during sharp-wave ripple complexes (O’Keefe, 1976; Buzsáki et al., 1983, 1992), a transient LFP pattern associating fast $(\sim 200 \mathrm{~Hz})$ oscillations in stratum pyramidale and a large deflection in stratum radiatum. These awake replay events have been involved in spatial working memory (Jadhav et al., 2012). Further replay of behavior-related neural sequences endogenously occurs during sleep (Wilson and McNaughton, 1994; Lee and Wilson, 2002), and has been shown to play a critical role in memory consolidation (Girardeau et al., 2009; Ego-Stengel and Wilson, 2010; Maingret et al., 2016; van de Ven et al., 2016).

Hippocampal sequences may thus represent a general neurophysiological mechanism supporting memory formation, consolidation and retrieval. The present review focuses on hippocampal theta sequences. We first describe the cellular and network mechanisms proposed to give rise to the thetarelated dynamics of hippocampal place cells. We then report evidence for a role in information processing and memory trace formation. Finally, we discuss possible functional distinctions between neural sequences emerging during theta and awake sharp-wave ripples.

\section{EMERGENCE OF THETA SEQUENCES}

The sequential activation of successive place cells along a given trajectory, at the behavioral timescale, does not trivially imply that in overlapping regions of space these same cells should fire in the same order at the much faster, "theta" timescale. How do theta sequences emerge in the hippocampal network? During locomotion, the hippocampal dynamics are under the combined influences of extrinsic inputs from multiple sensory and locomotion-related structures, as well as the intrinsic properties of the hippocampal network, modulated by elevated acetylcholine levels. Theta oscillations dominate the hippocampal LFP and powerfully shape both pyramidal cell and interneuron firing.

\subsection{Theta During Locomotion}

The theta rhythm includes a range of low and regular frequency oscillations from 4 to $12 \mathrm{~Hz}$ (Figure 1A). During wakefulness, theta oscillations arise during locomotion and epochs of active engagement in the environment such as navigating, exploring objects, rearing and preparing for movement (Jouvet, 1969; Vanderwolf, 1969; Foster et al., 1989). While the precise mechanisms responsible for the generation of theta remain to be elucidated (Buzsáki et al., 2002), theta oscillations are thought to result from an interplay between numerous factors (Figure 1B), including rhythmic cholinergic modulation and feed-forward inhibition from the medial septum and diagonal band of Broca (MSDB; Petsche et al., 1962; Kramis et al., 1975; Buzsáki et al., 1986; Vanderwolf et al., 1988; Kocsis et al., 1999; Müller and Remy, 2014; Zutshi et al., 2018), rhythmic inputs from layers II and III of the entorhinal cortex targeting DG/CA3 and CA1, respectively (Buzsáki et al., 1983; Kamondi et al., 1998), activation of CA1 pyramidal cell dendrites by CA3 collaterals (Konopacki et al., 1987; Kamondi et al., 1998; Fisher and Blum, 1999), interneuronal network dynamics within (Goutagny et al., 2009) and between (Jackson et al., 2014) hippocampal fields, rhythmic feedback inhibition from hippocampus long-range interneurons to MSDB (Alonso and Köhler, 1982; Tóth et al., 1993), as well as intrinsic resonant properties of hippocampal, entorhinal and medial septal neurons mediated by various voltage-dependent channels $\left(\mathrm{Ca}^{2+}, \mathrm{K}^{+}, \mathrm{Na}^{+}\right)$which provide membrane resonance and subthreshold oscillations at theta frequency (Kamondi et al., 1998; Evstratova et al., 2011; Hansen et al., 2014).

The theta rhythm appears to act as an internal clock: hippocampal pyramidal cells fire maximally at the trough of theta cycles, when perisomatic inhibition by parvalbumin (PV) and cholecystokinin (CCK) basket cells, as well as axo-axonic cells, is minimal; and different classes of GABAergic neurons have different preferred firing phases (Freund and Buzsáki, 1996; Csicsvari et al., 1999; Klausberger and Somogyi, 2008; Müller and Remy, 2014). Local GABAergic inhibition may fine tune the spike timing of pyramidal cells relative to theta [e.g., Ego-Stengel and Wilson, 2007; Geisler et al., 2007; Maurer and McNaughton, 2007; Royer et al., 2012; Grienberger et al., 2017; see the network model by Ferguson et al. (2015)]. Given the strong and reliable modulation of hippocampal activity by theta oscillations, behavior-related changes in the theta profile (e.g., spectral components) may play a crucial role in hippocampal dynamics.

Many structures activated during locomotion modulate theta (Vertes et al., 2004; Kiehn, 2016). Consistently, theta is primarily influenced by ambulatory signals, compared to e.g., optic flow or vestibular self-motion cues (Czurkó et al., 1999; Terrazas et al., 2005). Hence, numerous subcortical structures, which may not be directly involved in the actual generation of theta, have nonetheless been reported to modulate theta. These are part of the "synchronizing ascending system" (Vertes and Kocsis, 1997; Vertes et al., 2004; Orzeł-Gryglewska et al., 2015; Kiehn, 2016). Efferences of several subnuclei of the brainstem reticular formation and the mesencephalic locomotor region can trigger the emergence (onset, offset) and the regulation (power, frequency) of theta (Grastyán et al., 1965; Peck and Vanderwolf, 1991; Sinnamon, 1993; Vertes and Kocsis, 1997; 

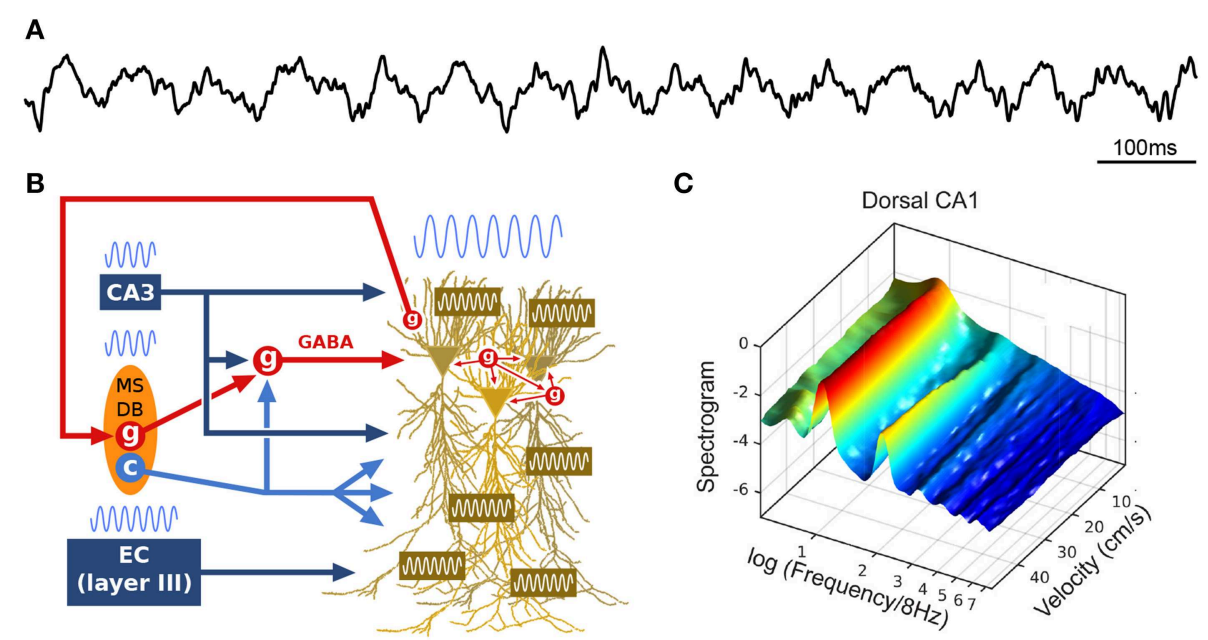

c

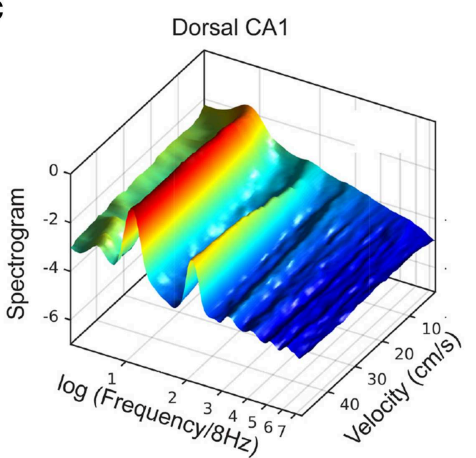

FIGURE 1 | Theta oscillations. (A) Example local field potential (LFP) trace during track running. (B) Simplified schematic of theta generation. The medial septum-diagonal band of Broca (MSDB) sends theta-rhythmic GABAergic (g), cholinergic (c) and glutamatergic projections to the hippocampus (glutamatergic neurons appear to entrain other septal neurons, but not hippocampal theta, and are not represented here). Rhythmic inhibition (red, GABA) of highly interconnected perisomatic interneurons (g) constrains the timing of pyramidal cell spikes. The entorhinal cortex (EC) also sends theta-paced outputs to the hippocampus through the perforant path. CA3 outputs targeting stratum radiatum constitute another likely intrahippocampal theta generator. In addition, the dendritic membrane of CA1 pyramidal cells is endowed with resonant properties at theta frequency. Finally, hippocampal long-range backpropagating interneurons target the MSDB, arguing for cyclical (non-unidirectional) mechanisms in the generation of theta. (C) Power spectral density of dorsal CA1 LFP as a function of running velocity (logarithmic scale; average over four rats). At low velocities, power is dominated by a single peak located between 6 and $7 \mathrm{~Hz}$. As velocity increases, the peak quickly narrows, shifts toward $8 \mathrm{~Hz}$, while additional peaks (harmonics) develop at frequencies $n \times 8 \mathrm{~Hz}$ (up to $n=6$ for dorsal CA1 region, $n=2$ for intermediate CA1). Reproduced from Sheremet et al. (2016).

Sńska and Kasicki, 1998; Jackson et al., 2008; Bland et al., 2016; Ma et al., 2017).

How is theta altered by locomotion? In the spectral domain, a $16 \mathrm{~Hz}$ oscillation, described as the second harmonic of theta (Harper, 1971), has been reported to emerge with increasing locomotion velocity (Figure 1C; Czurkó et al., 1999; Terrazas et al., 2005; Sheremet et al., 2016). More generally, a chain of harmonics significantly phase coupled to the theta oscillation appear to be related to the velocity of the animal (Sheremet et al., 2016). In the time domain, these speed-related nonlinearities are manifested as an increase in skewness and asymmetry in the shape of theta cycles (Buzsáki et al., 1983; Terrazas et al., 2005; Sheremet et al., 2016), which decrease along the septo-temporal axis of the hippocampus (Maurer et al., 2005; Hinman et al., 2011, 2013; Sheremet et al., 2016). In addition, the frequency of theta and its harmonics increase with running velocity (McFarland et al., 1975; Sńska and Kasicki, 1998; Maurer et al., 2005; Bender et al., 2015; Sheremet et al., 2016), in a consistent manner across the septo-temporal axis (Maurer et al., 2005; Sheremet et al., 2016). Intriguingly, theta phase shifts progressively from the septal to the temporal pole of the hippocampus, and thus theta can be seen as a "traveling wave" (Lubenov and Siapas, 2009; Patel et al., 2012).

Changes in theta power and frequency coincide with systematic changes in the spiking dynamics of pyramidal cells (Song et al., 2005b; Terrazas et al., 2005; Lu and Bilkey, 2010), and a strong correlation has been reported between theta nonlinearity and the intensity and timing of the spiking activity of both pyramidal cells and interneurons (Maurer et al., 2005; Sheremet et al., 2016). Thus, speed-related changes in theta oscillations have a strong impact on the precise spike timing of hippocampal cells. As described below, the precise phase at which spikes are emitted is directly related to the position of the animal in the environment (O'Keefe and Recce, 1993), which may constitute one of the clearest examples of temporal coding in the brain.

\subsection{Theta Phase Precession}

As a rat walks through the firing field of a given place cell, the firing rate of this cell progressively increases until the animal reaches the center of the place field, then decreases until the animal leaves the field. Thus, the firing rate of the place cell appears to code for the location of the animal in the environment. Strikingly, O'Keefe and Recce (1993) discovered that spatial information can also be provided by the precise time at which the place cell fires relative to the ongoing theta rhythm. Indeed, the place cell fires at progressively earlier phases across successive theta cycles: firing occurs at late phases when the animal enters the place field, then progressively shifts back in phase as the animal progresses through the field, and finally occurs at the beginning of the theta cycle when the animal leaves the field. This phenomenon is referred to as "phase precession" (Figure 2A; O'Keefe and Recce, 1993). While the range over which spike theta phase precesses may appear to span as much as $360^{\circ}$ when pooling data over multiple field crossings, single-trial phase range is typically $\sim 180^{\circ}$, and never exceeds a full cycle (O'Keefe and Recce, 1993; Maurer et al., 2006; Schmidt et al., 2009). At a given (fixed) running speed, the rate of phase precession is inversely related to the size of the place field: in particular, place cells with 

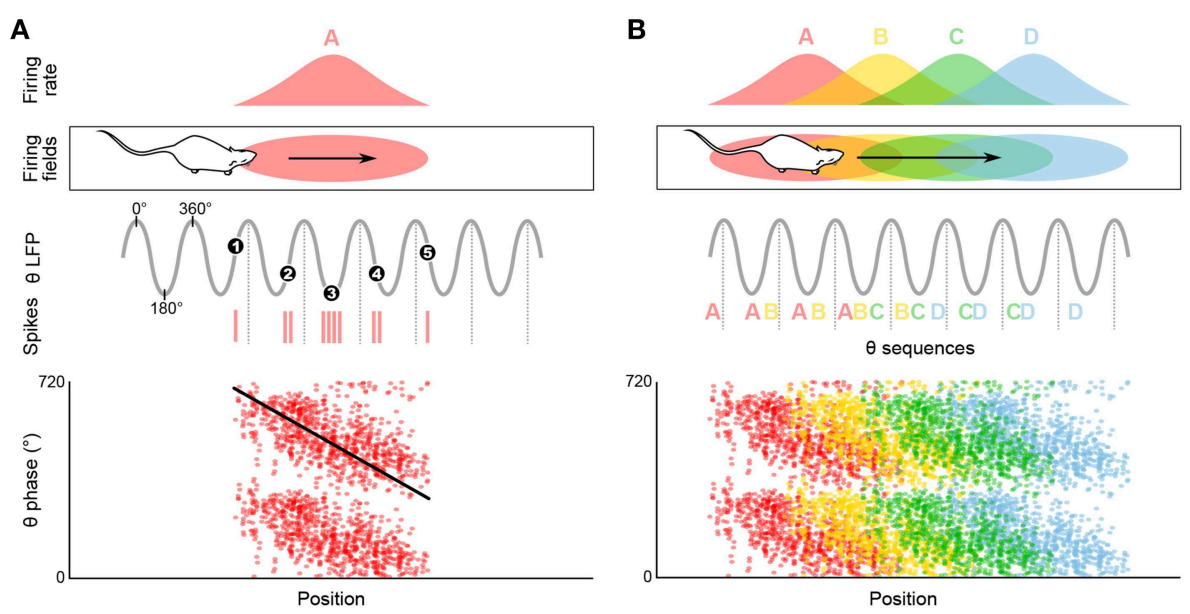

FIGURE 2 | Temporal coding in the hippocampus and emergence of theta sequences. (A) Theta spike phase precession. Top, schematic representation of the temporal organization of successive place cell bursts relative to theta as a rat runs along a linear track. When the rat enters the firing field (pink ellipse), the cell fires near the end of the theta cycle, i.e., with a phase of $\sim 360^{\circ}$. As the rat progresses through the field, bursts occur on earlier and earlier phases (red vertical ticks, action potentials; black numbered dots, mean burst times within individual theta cycles). Bottom, phase precession plot. For each spike, phase is represented as a function of position (tilted black line, best linear-circular regression line). (B) Top, four schematic overlapping firing fields. Middle, in their overlap region the cells discharge in sequence (A, $A B, A B C$, etc.) within each theta cycle, reflecting the order of field traversal at a compressed time-scale. Bottom, these past-present-future "sweeps" may require coordination between individual phase precessing cells.

small fields in the dorsal hippocampus phase precess faster than place cells with large fields in the ventral hippocampus (Maurer et al., 2005). On the other hand, phase precession slope increases with running speed, ensuring that the phase-position relationship is maintained regardless of running speed (Bose and Recce, 2001; Geisler et al., 2007).

Phase precession has been reported not only in all subfields of the hippocampus (Skaggs et al., 1996; Johnson et al., 2007; Mizuseki et al., 2009), but also in neighboring parahippocampal structures projecting to the hippocampus, including the subiculum (Kim et al., 2012) and medial entorhinal cortex (MEC, Hafting et al., 2008), as well as in downstream structures, such as the medial prefrontal cortex (Jones and Wilson, 2005) and ventral striatum (van der Meer and Redish, 2011). Understanding the mechanisms of hippocampal phase precession is thus expected to provide invaluable insights about a general coding scheme present in multiple brain areas (Harris, 2005; Malhotra et al., 2012). Recent evidence of phase precession in the human hippocampus (Qasim and Jacobs, 2016) further confirms that theta phase precession remains relevant beyond rodent species.

\subsubsection{Mechanisms of Phase Precession: Computational Models and Experimental Data}

The neurophysiological mechanisms of phase precession are difficult to explore experimentally, but have been extensively investigated through computational modeling (for reviews, see Maurer and McNaughton, 2007; Burgess and O'Keefe, 2011). While no model appears to fully account for all aspects of phase precession, a continued dialogue between experimental and modeling approaches has provided constraints on potential generation mechanisms. Below, the three main classes of models of phase precession are briefly reviewed and discussed in light of experimental data.

The detuned oscillators model was originally proposed by O'Keefe and Recce (1993) in their initial report of phase precession, and remains one of the most elegant hypotheses to date $^{1}$ (Figure 3A). This model considers a place cell that oscillates at a slightly faster frequency than theta. These two oscillators, synchronized at the beginning of the place field, slowly shift due to their frequency difference. Because the place cell fires at the peaks of its own membrane potential oscillation, i.e., its more depolarized states, this occurs earlier and earlier relative to the slower theta oscillation, which results in phase precession. In addition, the frequency of the membrane potential oscillation is assumed to be proportional to running speed, so that the phase advance of spikes reflects the distance traveled through the place field, rather than the time spent in the field. However, because the field size is maintained despite changes in running speed, it has been proposed that the frequency of both oscillators increased with running speed to compensate for the decrease in numbers of theta cycles at high running speed (Geisler et al., $2007,2010)$. The model requires neurons with cosine directional tuning, which have been identified in the hippocampus, medial septum and anterior thalamus (Welday et al., 2011). Similar cells were documented in entorhinal cortex (Krupic et al., 2012), although the finding remains controversial (Krupic et al., 2015; Navratilova et al., 2016).

The generation sites of these two oscillators has remained unresolved. Intrahippocampal oscillators under the influence

\footnotetext{
${ }^{1}$ Incidentally, in its "uncorrected" version, this model predicted that place cells should fire in regular spatial patterns long before the discovery of grid cells (see e.g., Lengyel et al. (2003) for an influential model which eliminates repeated fields).
} 


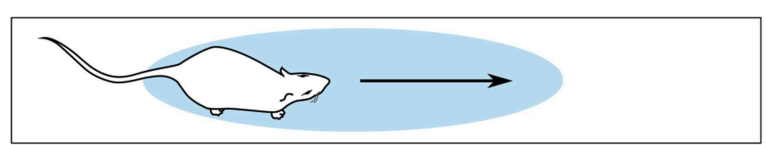

A

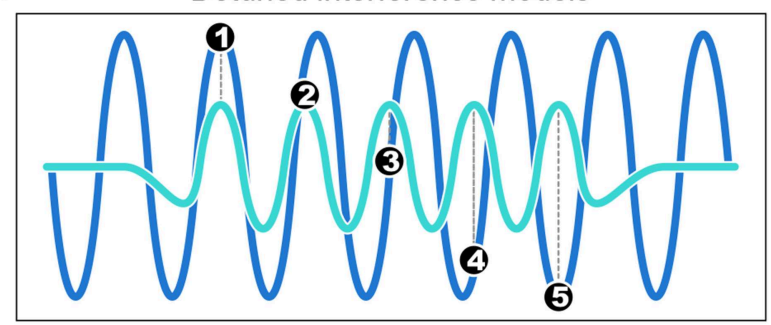

B

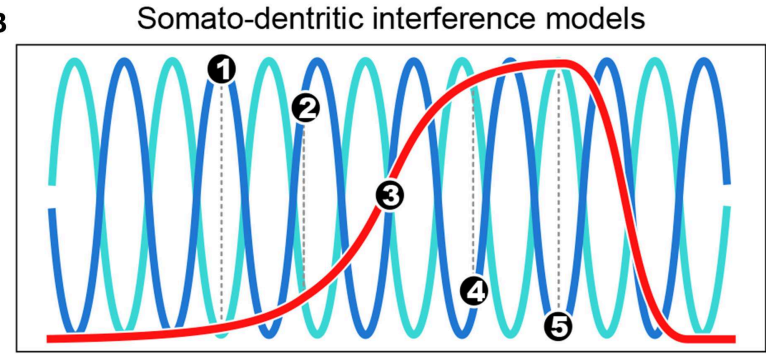

C

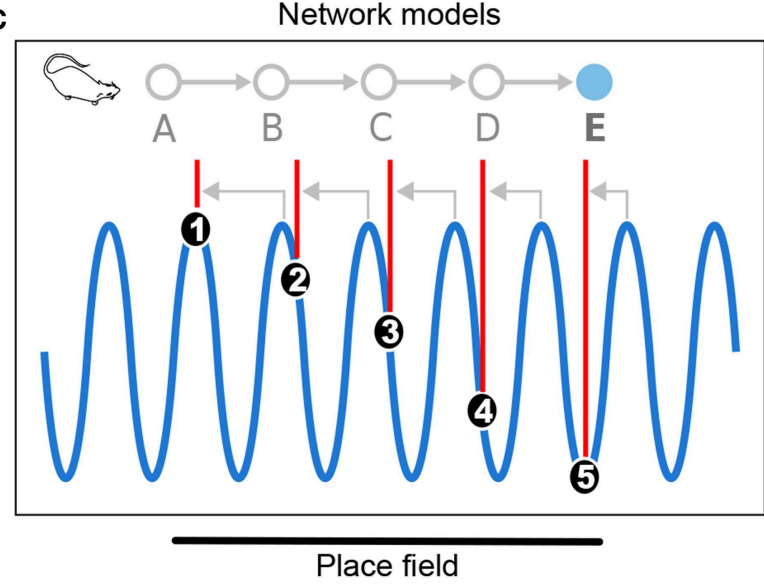

FIGURE 3 | Computational models of phase precession. (A) In the detuned oscillators models, the membrane potential $\left(V_{m}\right)$ of the neuron (light blue) oscillates faster than the local theta rhythm (dark blue) within the firing field. Spike bursts (black dots numbered from 1 to 5 ) occur at positive peaks of $V_{m}$ and thus anticipate theta cycles, resulting in phase precession. (B) In the somato-dendritic interference models with asymmetric excitation ramps, spikes are emitted whenever excitation (red) exceeds somatic inhibition (light blue), which is paced by theta oscillations (dark blue). Because excitation increases, it exceeds inhibition earlier and earlier, resulting in phase precession. (C) In the asymmetric connections model, successive place cells (labeled from A to E) with overlapping fields are connected (gray arrows) in such a way that activity propagates unidirectionally within the network. Thus, as the rat enters the field of cell $\mathrm{E}$, it happens to be located at the center of the field of cell A. Cell A is therefore maximally excited, and transmits its activity via the chain of intermediate cells until it reaches cell $\mathrm{E}$, which fires late in the theta cycle as a result of transmission delays. But as the rat progresses through the field, there are fewer and fewer intermediate steps and the transmission delays decrease, so that cell $\mathrm{E}$ fires earlier and earlier in the theta cycle. Note that in the present Figure, phase precession is shown from $360^{\circ}$ to $180^{\circ}$ to simplify the schematic representation. of medial septum and CA3 recurrent inputs are inconsistent with the finding that theta reset by brief single-pulse electrical stimulation of the ventral hippocampal commissure does not reinitialize phase precession and leaves the phase-position relationship intact (Zugaro et al., 2005). Extrahippocampal oscillators (e.g., Yamaguchi, 2003) would be supported by phase precession in MEC grid cells (in layer II and to a lesser extent in layer III) and conjunctive cells (in layer III) (Hafting et al., 2008; Reifenstein et al., 2012, 2014, 2016; Climer et al., 2013; Ebbesen et al., 2016), which persists during pharmacological inactivation of the hippocampus (Hafting et al., 2008). Conversely, hippocampal phase precession is disrupted after bilateral lesions of the MEC (Schlesiger et al., 2015, but see Ormond and McNaughton, 2015). Further, a recent study by Robinson et al. (2017) has shown that phase precession in object-responsive CA1 cells persists during transient optogenetic inactivation of the MEC, suggesting a possible complementary role for the LEC, which predominantly provides non-spatial information (Deshmukh and Knierim, 2011; Tsao et al., 2013). Several models have suggested that entorhinal oscillators might generate phase precession (Burgess et al., 2007; Climer et al., 2013; Jeewajee et al., 2014). On the other hand, relatively large phase shifts between EC and CA1/CA3 spikes (Mizuseki et al., 2009) remain at odds with the notion that phase precession could be directly transferred from the EC to the hippocampus. In addition, the mechanism proposed to account for phase precession in EC requires that entorhinal head direction cells signal movement direction, which is inconsistent with experimental findings (Cei et al., 2014; Raudies et al., 2015).

Somato-dendritic interference models (Figure 3B; Kamondi et al., 1998; Magee, 2001; Harris et al., 2002; Mehta et al., 2002; Losonczy et al., 2010) posit that the timing of action potentials is determined by the combination of oscillatory somatic inhibition and transient ramp-like dendritic excitation. As the animal enters the place field, the cell fires when excitation overcomes inhibition, which occurs earlier and earlier because of the ramplike shape of the dendritic depolarization. The excitation ramp has been proposed to be either symmetrical (Harris et al., 2002) or asymmetrical (Mehta et al., 2002). The asymmetrical depolarization shape mirrors the asymmetrical development of place field with experience (Mehta et al., 1997, 2000). To remain consistent with the observation that spike phase decreases but does not subsequently increase, the symmetric ramp model posits that the place cell stops firing at the peak of the ramp. This was supported by in vitro data obtained following symmetricalshaped depolarizing current injection through the pyramidal cell dendrites (Harris et al., 2002), which suggested that an adaptive mechanism allowed the cell to precisely stop firing at the ramp peak.

Both intracellular in vitro (Magee, 2001; Losonczy et al., 2010) and extracellular in vivo (Kamondi et al., 1998; Harris et al., 2002; Mehta et al., 2002) data are consistent with the somato-dentritic interference models. Notably, recordings of intracellular activity in awake behaving animals confirmed that within firing fields, the membrane potential of place cells had an asymmetric depolarization curve (Harvey et al., 2009; Epsztein et al., 2011; this was also observed in grid cells, Domnisoru 
et al., 2013; Schmidt-Hieber and Häusser, 2013). Epsztein et al. (2011) further documented an increase in the excitability of place cells during field traversal, compared to silent cells. While this supports the somato-dentritic interference models, the same studies also reported that spikes remain locked to the peaks of the membrane potential, a critical prediction of the detuned oscillators models. And because recent work has shown that even network connectivity models (see below) can account for these findings (Romani and Tsodyks, 2015), it becomes unclear whether intracellular data do discriminate between different classes of models of phase precession.

In the network connectivity models, contrary to the above cellular models in which no particular synaptic connectivity is assumed, phase precession results from transmission delays between asymmetrically connected cells (Figure 3C; Jensen and Lisman, 1996; Skaggs et al., 1996; Tsodyks et al., 1996; Romani and Tsodyks, 2015). According to these models, place cells are unidirectional and asymmetrically connected to each other. Feed-forward sensory inputs arriving when the animal reaches the end of the place field drive the cells to fire at early phases of the theta oscillation. Activity then spreads to place cells with fields located further along the trajectory (via asymmetric connections), which fire later in the cycle due to transmission delays. Hence, place cells fire earlier and earlier as the animal progresses through their field, resulting in phase precession. Asymmetric connections can be hard-wired (Dragoi and Tonegawa, 2011, 2013) or result from experience (Silva et al., 2015), consistent with the experience-dependent asymmetric expansion of place fields (Mehta et al., 1997, 2000). An alternative version of the model posits that phase precession results from phase differences between CA3 and entorhinal inputs (Chance, 2012; Fernández-Ruiz et al., 2017). In both cases, the proposed mechanisms are difficult to reconcile with the experimental finding that the relation between phase and allocentric spatial position changes during backward movement (Cei et al., 2014; Maurer et al., 2014). Further, this change occurs abruptly, ruling out the possibility that asymmetry could reverse via slow plasticity processes (Cei et al., 2014). Hence, these models may need refining to account for these experimental findings (but see Chadwick et al., 2015, 2016).

While phase precession has been mainly described in principal cells, there have been reports of phase precession in hippocampal interneurons, in particular those receiving putative monosynaptic connections from place cells recorded on the same tetrode. Within the field of their partner place cells, these interneurons share the same rate of phase precession (Maurer et al., 2006; Ego-Stengel and Wilson, 2007; Geisler et al., 2007). Because pyramidal cells generally fire before interneurons, the spatial selectivity of interneurons was suggested to be inherited from local pyramidal cells (but see Chadwick et al., 2016). Functionally, these interneurons could inhibit competing cell assemblies and therefore participate in the segregation of cell assemblies in space and time (Maurer et al., 2006; Cutsuridis and Hasselmo, 2012; Royer et al., 2012; Dupret et al., 2013). This is supported by recent data by Grienberger et al. (2017), who coupled LFP and intracellular recordings in head-restrained mice running on an enriched treadmill, and showed that optogenetic hyperpolarization of CA1 interneurons reduces both the rate and temporal coding of spatial location in CA1 place cells by suppressing out-of-field excitatory inputs.

\subsubsection{Functional Role of Phase Precession}

While phase precession may code for allocentric position on linear tracks (O'Keefe and Recce, 1993; Jensen and Lisman, 2000), it is unclear how this code would generalize to two dimensional environments. Contrary to linear tracks, in open fields animals can enter place fields from multiple directions, and place cells precess in a similar manner regardless of the direction of motion. As a result, place cells fire at the same theta phase for multiple locations (Skaggs et al., 1996; Huxter et al., 2008). Thus, phase may instead code for the relative distance from the place field center (Jeewajee et al., 2014). Similarly, the reversal of the phaseposition relation observed during backward travel (Cei et al., 2014; Maurer et al., 2014) indicates that phase does not code for the allocentric position of the animal, but more likely for the distance traveled through the field.

Phase precession has been observed in a variety of contexts including running on a wheel (Hirase et al., 1999; Pastalkova et al., 2008) or a treadmill (Royer et al., 2012; Cei et al., 2014; Grienberger et al., 2017), jumping (Lenck-Santini and Holmes, 2008), in virtual reality (Chen et al., 2013; Ravassard et al., 2013; Aghajan et al., 2015), and during fixation (Takahashi et al., 2014). While all of these conditions correspond to theta states, the animal does not necessarily change location. Consistently, several studies have reported object- and sound-modulated CA1 phase-precessing cells (Aronov et al., 2017; Robinson et al., 2017) and phase-precessing cells toward reward locations in the ventral striatum (van der Meer and Redish, 2011). This suggests that phase precession may not be uniquely linked to space, but rather to the full episodic component of the ongoing experience (e.g., reviewed in Jaramillo and Kempter, 2017). An even more drastic departure from the spatial coding hypothesis is the view that perhaps the main role of theta phase precession is the formation of neuronal activity sequences, segregated by theta cycles (Figure 2B; e.g., Skaggs et al., 1996; Schmidt et al., 2009).

\subsection{Mechanisms of Theta Sequences}

Whether phase precession is a cause or a consequence of the formation of theta sequences remains unresolved. As discussed above, phase precession could result from a variety of mechanisms that do not require sequential connectivity between neighboring place cells (detuned oscillators and somatodendritic interference models). Independent initiation of phase precession in successive cells would automatically yield theta sequences (Skaggs et al., 1996). Thus, phase precession would be a causal mechanism of theta sequences. An alternative view is that hippocampal sequences result from specific patterns of connectivity within the hippocampal network (network connectivity models; see also Dragoi and Tonegawa, 2011). At the single cell level, this would be manifested as phase precessing spikes. Thus, phase precession would be a consequence of the formation of theta sequences.

Several studies have challenged the idea that phase precession could entirely account for theta sequences, by showing that a 
moderate addition of time jitter to experimental spike trains resulted in preservation of phase precession but a reduced prevalence of theta sequences (Dragoi and Buzsáki, 2006; Foster and Wilson, 2007; Itskov et al., 2008). This suggested that theta sequences required precise coordination between cell assemblies in addition to independent phase precession mechanisms. Two recent studies have provided further support for this view. Feng et al. (2015) analyzed single-trial phase precession and theta sequence formation in rats exploring a novel linear track, and reported that while single place cells exhibit phase precession from the first lap, theta sequences do not emerge until the second lap. Middleton and McHugh (2016) analyzed the temporal coding of CA1 and CA3 neurons during spatial navigation in a transgenic mouse model lacking CA3 synaptic transmission (CA3 tetanus toxin mouse line). They showed that the absence of CA3 inputs abolished the formation of theta sequences at the CA1 ensemble level despite the persistence of phase precession in individual neurons. They suggested that CA3 may be instrumental for temporal coordination of the CA1 neuronal population. In summary, the above studies point to an intermediate mechanism, where phase precession plays an important but non-exclusive role in generating theta sequences, complemented by temporal coordination processes which may require specific functional connectivity.

To formally test the requirement for additional coordination mechanisms, Chadwick et al. (2015) developed phenomenological models of independent and coordinated place cell activity during navigation, and compared their predictions in light of experimental data. They concluded that the independent coding model is sufficient to replicate the data previously interpreted as evidence for the coordinated assembly hypothesis. First, although it has been shown that predictions of individual CA1 place cell spike timing is improved by information conveyed by their peer cells (Harris et al., 2003), suggesting that these cells directly interact with each other consistent with the coordinated assembly hypothesis (Harris, 2005), Chadwick et al. (2015) showed that this no longer holds when both the modulation of firing phase by travel direction (Huxter et al., 2008; Climer et al., 2013; Cei et al., 2014; Jeewajee et al., 2014; Maurer et al., 2014) and the modulation of firing rate by running speed (e.g., Czurkó et al., 1999; Huxter et al., 2003; Geisler et al., 2007) are taken into account to predict the activity of a given cell. As a matter of fact, peer information even decreases the average prediction, consistent with the independent coding model. Second, the independent coding model also replicated the finding that spike jitter can degrade theta sequences but spare the phase-position relation (Dragoi and Buzsáki, 2006; Foster and Wilson, 2007; Itskov et al., 2008), and the covariance in firing rate between cell pairs across successive running laps (Foster and Wilson, 2007). Finally, the independent coding model also accounted for global remapping data that the coordinated assembly model failed to replicate.

Incidentally, both the independent coding and coordinated assembly models can account for internal sequence generation (Pastalkova et al., 2008; Wang et al., 2015), proposed to support cognitive processes. One potential issue with the independent coding model, which is not directly related to the generation of theta sequences, is that it remains unclear how sequences could reactivate during non-theta states (see also Chadwick et al., 2016). A possible solution would be that once theta sequences emerge due to independent phase precession, the repeated activation of successive neurons induces synaptic changes that result in asymmetric network connectivity; this would allow trajectory replay during sleep (Drieu et al., 2018; see section 3.3).

While direct interactions between pyramidal neurons may not be required, specific classes of hippocampal interneurons may be involved in coordinating sequential information. As stated earlier, despite the fact that interneuron activity usually displays weak spatial modulation (McNaughton et al., 1983), many interneurons fire spikes that precess in phase against the theta rhythm, tightly coupled to monosynaptically connected pyramidal cells (Maurer et al., 2006; Ego-Stengel and Wilson, 2007; Geisler et al., 2007). While this suggested that interneuron phase precession may be inherited from place cells (Maurer et al., 2006; Geisler et al., 2007), Chadwick et al. (2016) recently proposed an alternative model of theta sequences where phase precessing interneurons play a crucial role in coordination of place cell spike timing and the emergence of theta sequences. In their model, phase precession occurs dynamically whenever a place cell is driven by external inputs due to the transient functional coupling between place cells and interneurons. Consequently, phase precession and theta sequences are generated de novo within the network, and slow input sequences are automatically compressed into theta sequences in networks of interacting pyramidal cells and interneurons. This is consistent with the findings of Royer et al. (2012), who reported that optogenetic silencing of PV-expressing interneurons shifted the phase of pyramidal cell spikes toward the trough of theta (conversely, silencing of SOM-expressing interneurons increased the burstiness of pyramidal cell spikes without altering their theta phase). This suggests that somatic inhibition may play a major role in theta phase precession and segregation of cell assemblies (Royer et al., 2012; Dupret et al., 2013; Chadwick et al., 2016).

\section{THETA SEQUENCES, NAVIGATION, AND MEMORY}

Both theoretical considerations and experimental data indicate that theta phase precession and theta sequences could underlie cognitive functions. While the seminal report of O'Keefe and Recce (1993) emphasized the probable role of phase precession in spatial coding, the authors also immediately recognized that phase precession placed conditions on the possibility that synaptic connections could be modified. This was shortly followed by suggestions that a critical function of phase precession was to bring neurons to fire within brief time windows conducive to synaptic plasticity (Burgess et al., 1994; Lisman and Idiart, 1995; Skaggs et al., 1996). Effectively, theta sequences would allow the hippocampal network to store temporally ordered information, such as events in their spatio-temporal context, thus constituting a substrate for episodic-like memory trace formation. Consistently, there is evidence that degradation 
of theta sequences impairs sleep replay underlying memory consolidation (Drieu et al., 2018), and is accompanied by memory deficits (Lenck-Santini et al., 2008; Robbe and Buzsáki, 2009). In addition, recent data indicate that theta sequences reflect ongoing cognitive processes (Wikenheiser and Redish, 2015b), suggesting that they could also be involved in planning and decision making.

\subsection{Theta-Gamma Neural Code}

During theta states, nested gamma oscillations shape the activity of cell assemblies so that largely non-overlapping ensembles are sequentially activated in the $10-30 \mathrm{~ms}$ time windows of successive gamma cycles which occur at successive theta phases (Lisman and Idiart, 1995; Harris et al., 2003; Harris, 2005). This would enable the hippocampal network to represent four to eight items per theta cycle (corresponding to the number of nested gamma cycles), which has been related (Lisman and Idiart, 1995) to the "magic number" of $7 \pm 2$ items that humans can typically hold in short term memory (Miller, 1956). Hence, theta-gamma coupling has been hypothesized to form a neural code representing multiple items in a temporal frame (Figure 4A; Jensen and Lisman, 2005; Lisman and Buzsáki, 2008; Lisman and Jensen, 2013). Buzsáki (2010) even proposes the concept of a neural syntax, in which cell assemblies, the fundamental computational unit (letters), are arranged in specific orders by oscillations, external or internal excitatory inputs and local inhibition, in order to form words.

Evidence for a role of theta in information chunking has been provided in a number of experimental studies. By quickly changing light cues on the walls and floor of a recording chamber, Jezek et al. (2011) induced rapid switches between two perceived environments, and reported that $\mathrm{CA} 3$ representations flickered between the two maps. These network flickers tended to occur at the timescale of one or a few theta cycles. Notably, activity within a single theta cycle correlated with either environment, but rarely with both simultaneously, suggesting that theta cycles constituted temporal units of information. Similarly, Gupta et al. (2012) recorded CA1 activity while rats performed a decision task on a eight-shape maze with two reward sites, and reported that neural sequences expressed during single theta cycles reflected cognitive segments of the task. This supported the view that theta cycles may underlie the cognitive chunking of experience. Further, they reported that the length of the path encoded in a given theta cycle was directly related to the length of the cycle and to the number of gamma cycles it contained, suggesting that gamma cycles constitute information processing steps (see also Zheng et al., 2016).

The role of gamma oscillations in organizing spike timing has been supported by several recent studies. During theta oscillations, at least two types of nested gamma oscillations can occur in short bursts (Bragin et al., 1995), although it remains unclear whether these can occur within single theta cycles (Belluscio et al., 2012) or they are restricted to different theta cycles (Colgin et al., 2009). In CA1, slow gamma (gammas, $\sim 30-50 \mathrm{~Hz}$ ) preferentially occurs on the descending phase of theta. It is thought to arise from $\mathrm{CA} 3$ and to propagate along the Schaffer collaterals to the stratum radiatum. Medium gamma oscillations (gamma,$\sim 50-90 \mathrm{~Hz}$ ) tend to occur near the theta peak and may emerge from MEC inputs targeting the stratum lacunosum-moleculare (Bragin et al., 1995; Csicsvari et al., 2003; Colgin et al., 2009; Belluscio et al., 2012; Schomburg et al., 2014; Buzsáki and Schomburg, 2015).

Takahashi et al. (2014) studied hippocampal responses during fixation periods in a delayed spatial-alternation task. Neurons selective for the alternation sequence phase precessed during the entire fixation period, shifting their spikes from the peak to the trough of theta oscillations. Concomitantly, gamma power (60$90 \mathrm{~Hz}$ ) arising at the theta peak decreased, while gammas power $(30-45 \mathrm{~Hz})$ arising at the theta trough increased toward the end of the fixation period. The authors hypothesized that CA1 received predominant inputs from the EC at fixation onset, providing information about ongoing context. This signal would then elicit auto-associative intra-hippocampal processing in CA3 to recall the required action, or to look up in memory the appropriate task sequence given the ongoing context. This scenario, reminiscent of the model of Chance (2012), is consistent with the finding that gammas power is reduced and theta sequences are disrupted in inducible KO mice lacking CA3 inputs (Middleton and McHugh, 2016). Different gamma frequency bands would thus reflect different types of information processing, e.g., externally cued vs. internally generated.

This is in line with the notion that theta cycles have two complementary functional modes (Hasselmo et al., 2002): an encoding phase at the peak of theta (as recorded from CA1 pyramidal layer), largely mediated by EC inputs, and a retrieval phase at the theta trough mediated by CA3 recurrent activity. Incidentally, this could explain why Jezek et al. (2011) reported a better separation of the two hippocampal maps during the second half of the theta cycle, when CA3 retrieval mode would dominate. A similar dissociation would be expected in the study of Gupta et al. (2012), where theta sequences representing trajectories ahead of the animal, reflecting memory retrieval, could be linked to higher gammas band power, whereas trajectories behind the animal, reflecting memory encoding, could be linked to higher gammam band power. Unfortunately, this was not investigated, but two recent studies reported a similar dissociation in rats running on a linear track.

In the first study, Bieri et al. (2014) found that late theta phases were dominated by gammas, when spikes appear to anticipate the upcoming field location ("prospective" firing, Battaglia et al., 2004). In contrast, early phases were dominated by gamma $\mathrm{M}_{\mathrm{M}}$, when spikes lag behind field location ("retrospective" firing). These results suggest that alternating gammas and gamma states allow the hippocampus to switch between prospective and retrospective modes, possibly to prevent interference between memory retrieval and encoding.

In the second study, Zheng et al. (2016) performed Bayesian reconstruction of the trajectory of the animals at the theta timescale, and showed that theta sequences more accurately represented ongoing trajectories during theta cycles dominated by gamma $a_{M}$ than during theta cycles dominated by gammas (Figure 4B). Gammas-associated theta sequences were found instead to represented relatively long paths extending ahead of the current location. In these sequences, spike phases relative 


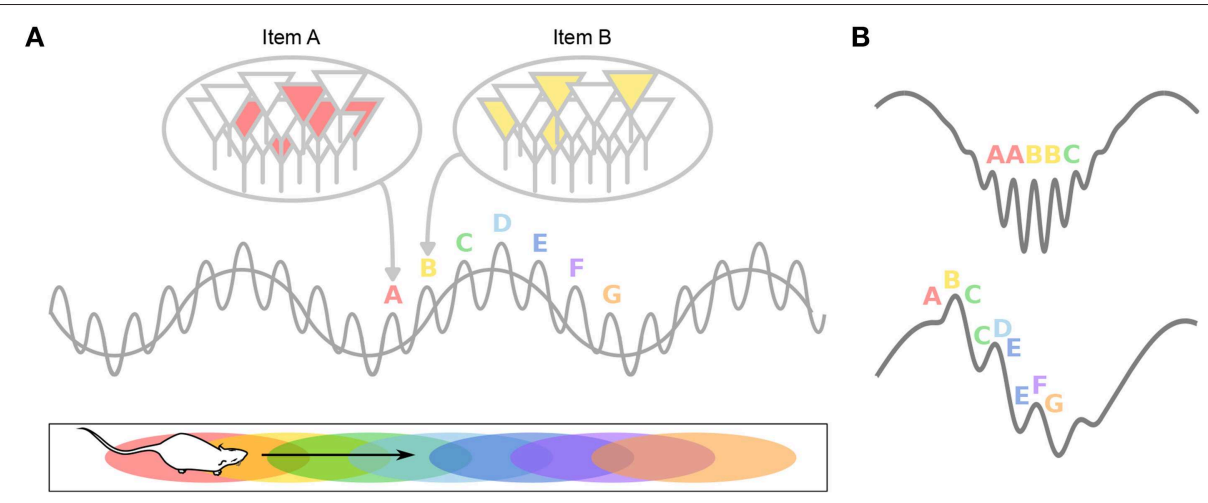

FIGURE 4 | Theta-gamma neural code. (A) Individual memory representations (cell assemblies, identified by letters) are repeatedly activated within each new theta cycle. The gamma rhythm separates the respective representations in time. The number of gamma cycles per theta cycle determines the span of working memory. (B) If discrete locations (i.e., A-G) are encoded within individual gamma cycles, longer paths would be encoded during fast gamma than during slow gamma. Alternatively, slow (but not fast) gamma cycles encode sequences of locations, rather than single locations. This results in longer paths encoded during slow gamma than during fast gamma.

to gammas also conveyed spatial information (Senior et al., 2008). This again argues that encoding and retrieval could be mediated by different gamma subtypes: relatively long gammas periods may allow successive items within the stored sequence to be retrieved in a time-compressed manner; in contrast, short periods of gamma ${ }_{M}$ would encode spatial memories in real time and may ensure chunking of cell assemblies. Note however that in this study, entire theta cycles were considered to contribute either gammas or gamma ${ }_{M}$ sequences (depending on the overall dominating gamma band), but not both (although see e.g., Belluscio et al., 2012), leaving unexplored the possibility that encoding and retrieval could both occur within single theta cycles (Hasselmo et al., 2002).

The spatial strategy of the animal may also bias the balance

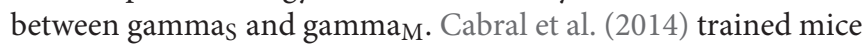
to find food rewards in a five-arm maze. During training, mice always started exploring the maze from the same location. But during test trials, they started from a different location, which allowed to assess if the animals used a place strategy (navigation to the same place in space) vs. a sequence strategy (performing the same series of left or right turns). When the mice used a sequence strategy, gammas was most prominent and place maps were more similar to training trials (after realignment), compared to when the mice used a place strategy, when spikes emitted during gammas and gamma $a_{M}$ periods contributed equally to the hippocampal representation. A possible interpretation is that during sequence strategy trials, the hippocampal network retrieves sequences of cell assemblies stored in CA3, while during place strategy trials, hippocampal activity would be equally driven by the recall of memory stored in CA3 and by landmark-related sensory information conveyed by the EC.

\subsection{Theta Sequences and Trajectory Planning}

As hinted previously, theta sequences do not appear to be entirely driven by sensori-motor signals. For instance, Gupta et al. (2012) reported that "look-ahead" or "look-behind" theta sweeps were preferentially expressed in different maze segments, and were modulated by the running velocity of the animal (Figure 5). Bieri et al. (2014) found that prospective spikes tended to occur when rats were leaving reward locations (the ends of a linear track), whereas retrospective spikes tended to occur as rats approached reward locations.

Convincing and direct evidence that cognitive mechanisms can regulate theta sequences was provided by Johnson et al. (2007), who recorded CA3 activity in rats performing two different versions of the T-maze decision task. At decision points, when the rat had to choose to turn left or right, theta sequences quickly and repeatedly swept forward toward the reward sites, alternatively representing the two possible paths. Similar anticipatory sequences occurred upon recovery from an error (Figure 5). The animals performed two different tasks: a multiple-T task, in which reward locations were fixed within a given session but changed from day to day, and a cued-choice task, in which a tone indicated for each lap which arm would be rewarded. In the multiple-T task, the number of sweeps down the unrewarded arm decreased across trials. In contrast, in the cuedchoice task, sweeps kept representing both directions throughout the session. Interestingly, at the decision point, the rats often expressed a typical behavior, consisting in pausing and orienting the head toward the left or right corridors, as if deliberating over the choice. This process is referred to as "vicarious trial and error" (VTE; Muenzinger and Gentry, 1931). Subsequent studies further linked VTE to decision-making processes (Schmidt et al., 2013; Amemiya and Redish, 2016; for a complete review see Redish, 2016). Of particular note, Wikenheiser and Redish (2015b) showed that theta sequences predicted longer trajectories when the animal was actually planning to reach a more distant goal. Thus, theta sequences also convey information about ongoing goals or intentions.

\subsection{Theta Sequences and Memory}

Because the mechanisms underlying theta sequences remain poorly understood and may involve many interacting factors 

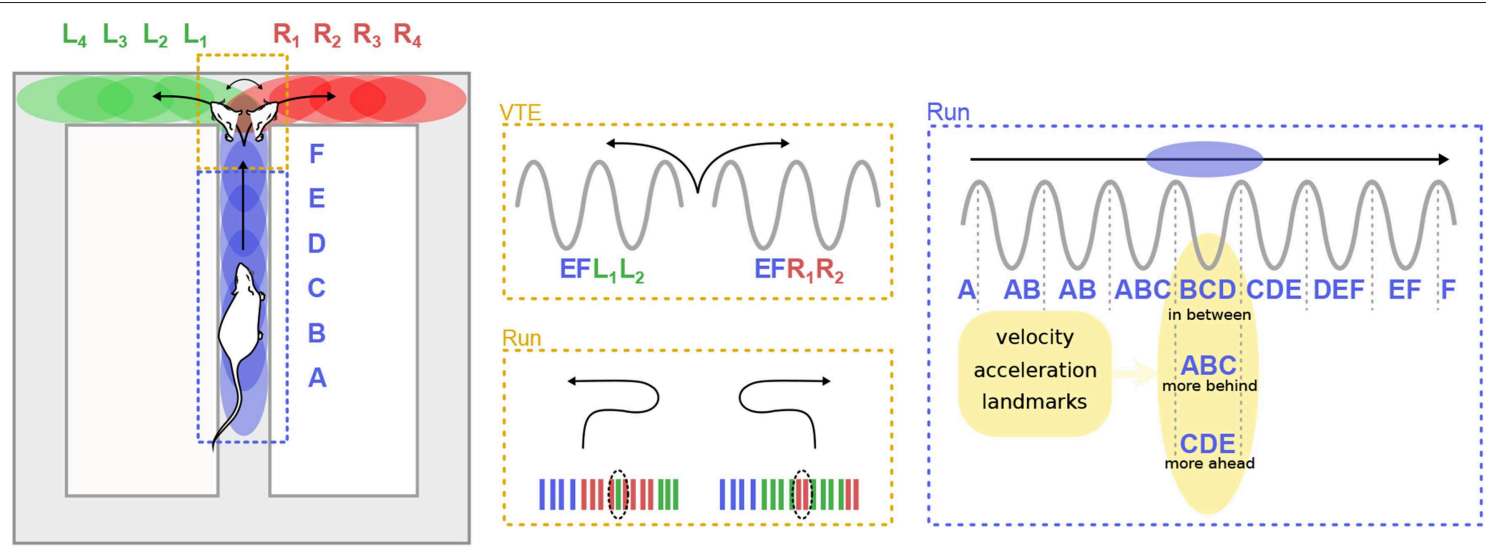

FIGURE 5 | Theta sequences and trajectory planning. (Left) A rat runs on the central arm of a maze (blue dashed box) and must decide whether to turn left of right at a choice point (red dashed box). Letters represent hippocampal cell assemblies and colored ellipses illustrate their respective firing fields on the maze. Center. During deliberation (vicarious trial and error, VTE) at the choice point, sequences of cell assemblies alternatively sweep toward the two possible paths (top). Extra-field firing can occur before the animal turns back toward the choice point during error correction trials (bottom). (Right) When the animal runs along a segment of the maze, theta sequences can represent positions centered on the animal ("in-between"), behind the animal ("more behind") or ahead ("more ahead"). The spatial representation embedded in theta sequences is influenced by multiple factors, including the velocity and acceleration of the animal, as well as the location of surrounding landmarks.

(including single-cell properties, synaptic connectivity patterns, local inhibition, theta-paced inputs, etc.), interfering with theta sequences is challenging. To the best of our knowledge, only three studies have reported task performance deficits linked to a degradation of theta sequences.

In the first study, Lenck-Santini et al. (2008) investigated hippocampal theta dynamics in rats, following status epilepticus, a condition that leads to mesial temporal lobe epilepsy (MTLE). These rats had altered phase precession and temporal organization of firing among pairs of neurons, along with deficits in spatial learning in the Morris water maze. This suggests that memory impairments stemmed from impaired temporal coding, consistent with a role for theta sequences in normal memory function.

In the second study, Robbe and Buzsáki (2009) examined the involvement of cannabinoid receptors in the dynamics of the hippocampal network (see also Robbe et al., 2006). Rats were trained to perform a hippocampus-dependent delayed spatial alternation task (Figure 6A). Administration of the cannabinoid receptor agonist CP55940 profoundly and reversibly impaired task performance. While the hippocampal place representation remained essentially intact, both theta phase precession and spike-timing coordination between place cells at the theta timescale were reversibly reduced. Notably, the percentage of correct trials on the task was correlated with the theta-scale pairwise coordination (assessed as the "compression index," i.e., the ratio of the distance between the place fields of pairs of place cells and the average time lag between their spike trains at the theta timescale). These results strongly argue for a role of phase precession and theta sequences in task performance. One potential caveat however is that CP55940 administration decreases ripple power (Robbe et al., 2006). Since place cell activity during awake ripples may play a crucial role in working memory (Jadhav et al., 2012), it is conceivable that the performance deficit could also result from altered place cell activity during awake ripples.

Wang et al. (2015) also reported impaired task performance following perturbation of theta-timescale activity (Figure 6B). In rats performing a delayed spatial alternation task, medial septum inactivation by muscimol injection abolished theta sequences both on the maze and in the wheel where the rats ran during delay periods (although measures of theta cycles and therefore theta sequences may not be as reliable when theta amplitude vanishes following muscimol injection). On the other hand, the placecell representation was spared when the rats were navigating the maze. Yet, performance was impaired. This suggests that theta sequences normally formed on the maze would have been necessary to achieve optimal levels of performance. One cannot entirely rule out that the observed performance deficits could be directly due to disruption of theta oscillations (Pan and McNaughton, 1997; Hasselmo et al., 2002) induced by medial septum inactivation $(\sim 80 \%$ decrease in theta power, Wang et al., 2015).

In summary, the above studies are consistent with the view that theta sequences are involved in the formation and recall of spatial memory, possibly by enabling the synaptic changes that underlie the storage of initial memory traces (Burgess et al., 1994; Skaggs et al., 1996). Yet, an alternative possibility is that these performance deficits resulted from impairments in planning and decision making, which also appear to involve theta sequences (Johnson et al., 2007; Schmidt et al., 2013; Wikenheiser and Redish, 2015b; Amemiya and Redish, 2016), rather than from deterioration of learning and memory processes. Thus, are theta sequences actually necessary for memory formation and recall? Bittner et al. (2017) recently uncovered a novel form of synaptic potentiation occurring at the behavioral timescale, and proposed that this could suffice to store entire sequences of events that took place several seconds before and after initiation 
A
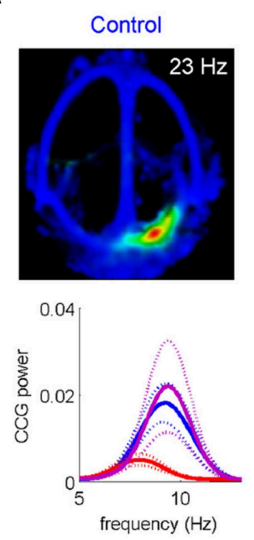
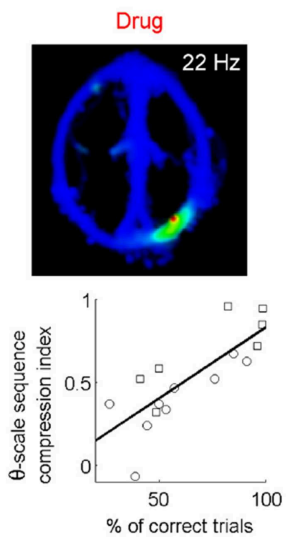
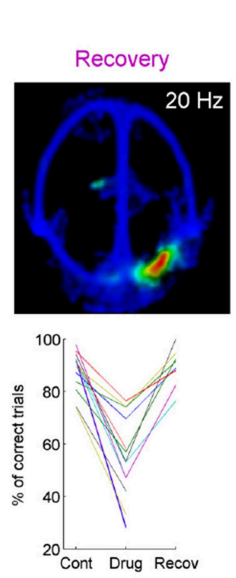

B
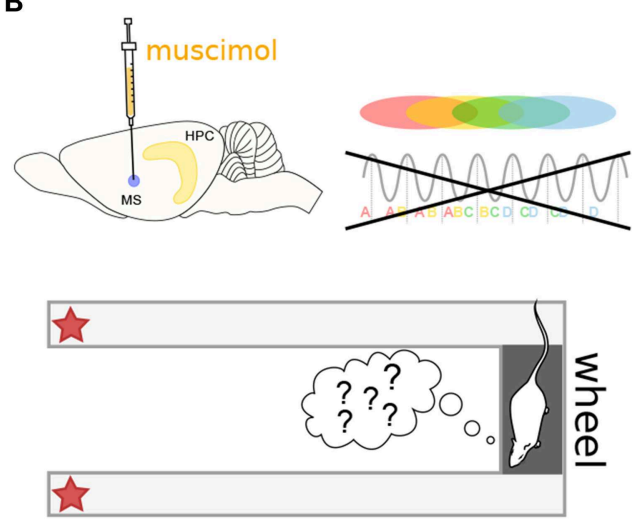

C
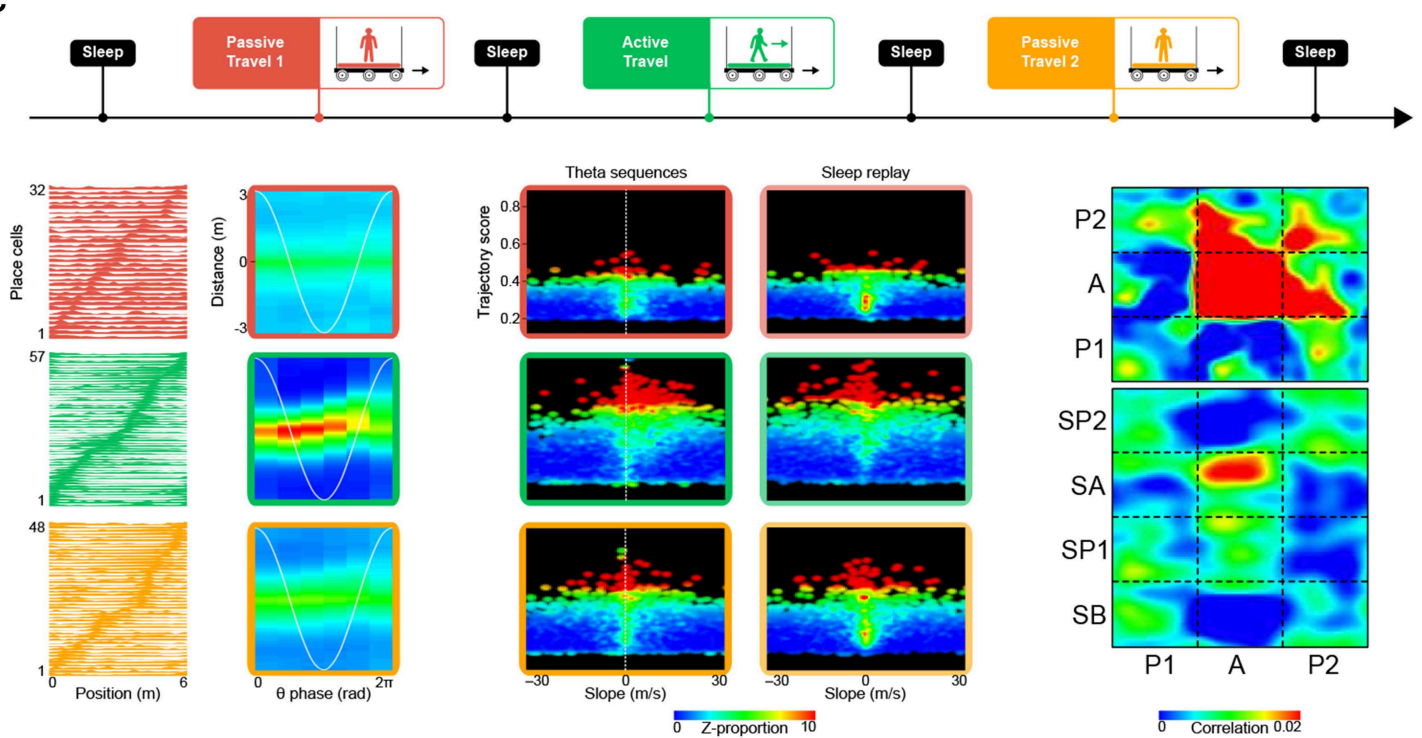

FIGURE 6 | Theta sequences and memory. (A) Selective perturbation of phase precession, but not firing maps, by injection of cannabinoid receptor agonist CP55940 induces performance deficits in a spatial memory task. Top. Firing maps for an example place cell recorded in control (left), drug (center), and recovery (right) conditions. Bottom left. Average power of the cross-correlograms for all neuron pairs with overlapping fields during control (blue), drug (red), and recovery conditions (magenta; dotted lines: s.e.m.) Bottom center. Correlation between behavioral performance (percentage of correct trials) and theta-scale sequence compression index in one rat. The different symbols refer to data obtained in two different experiments. Bottom right. Average performance (correct trials) for all sessions and rats.

(B) Medial septum inactivation by muscimol infusion impairs theta sequences and behavioral performance. (C) Perturbation of theta sequences impairs subsequent sleep replay. Top. Experimental protocol. The rats traveled on a model train. In successive sessions, a miniature treadmill was either turned off (passive travel; red and yellow) or on (active travel; green) to selectively disrupt or maintain theta sequences, while leaving behavioral timescale sequences intact. Bottom left. Firing fields (left column) remained stable in all conditions (red, green and yellow curves), but theta sequences (right column) emerged only during active travel (green box). Bottom center. Trajectories represented by hippocampal sequences were assessed using a combination of measures, namely trajectory score (linearity) and slope (velocity). During passive travel and subsequent sleep (red and yellow boxes), a very low proportion of both theta and replay sequences emerged in the hippocampal network (upper right quadrants are mostly black). On the contrary, during active travel and subsequent sleep (green boxes), a dramatically higher number of theta sequences and replay events were observed (red dots in upper right quadrants). Bottom right. Similarity of theta and replay sequences (P1, P2: passive travel sessions; A: active travel session; SA, SP1, SP2: sleep following respective travel sessions). Theta sequences were clear and self-consistent only during active travel (red zone at [A,A]). Sleep replay mirrored theta sequences only following active travel (red zone at [A,SA]). Panel (A) adapted from Robbe and Buzsáki (2009); panel (C) adapted from Drieu et al. (2018), with permission from AAAS.

of plateau-potentials in CA1 pyramidal cells. This effectively undermines the theoretical framework that theta sequences are required to bring spike trains in close temporal proximity and enable synaptic potentiation, underlying hippocampusdependent memory encoding.
Because during exploration, theta sequences are nested within behavioral timescale sequences, disentangling their respective roles in memory encoding requires a protocol that instantly and selectively blocks or releases theta sequences throughout the hippocampal system, but spares the firing fields of place cells 
(and therefore, behavioral timescale sequences). Our previous work has established that passive travel alters phase precession in most place cells (see also Song et al., 2005a; Terrazas et al., 2005), except if the rats actively run on a treadmill while being transported (Cei et al., 2014). Importantly, switching the treadmill on or off does not induce global remapping of place cells. We have recently leveraged this protocol to selectively impair phase precession (treadmill off) or leave it intact (treadmill on), while maintaining the spatial layout of place fields in the environment (Drieu et al., 2018). The rats were tested in an entirely novel environment, to stimulate spatial learning, network coordination and synaptic plasticity (Li et al., 2003; Leutgeb et al., 2004; Cheng and Frank, 2008; O’Neill et al., 2008), as well as to ensure that previously formed memory traces would not bias our observations. As expected, impaired phase precession during passive travel (treadmill off) was accompanied by an almost complete absence of theta sequences. On the other hand, behavioral timescale sequences were unaffected. During subsequent sleep, the hippocampal network failed to replay trajectories. Only following "active" travel (treadmill on), when theta sequences emerged nested within behavioral timescale sequences, did the hippocampal network generate genuine replay events during sleep (Drieu et al., 2018) (Figure 6C). This indicates that theta sequences play a causal role in establishing initial memory traces, which can later be reinstated during sleep for memory consolidation.

Note that in this study, the functional role of theta sequences was not assessed from behavior (task performance), but from the occurrence of dynamic network patterns with a welldocumented function, namely trajectory replay underlying memory consolidation. As a consequence, this alleviates the ambiguity discussed earlier, that behavioral impairments could result either from memory deficits, or from impairments in planning and decision making.

Interestingly, additional evidence for a link between theta sequences and sleep replay was recently provided by Muessig et al. (2019), who recorded from rat pups at different postnatal stages. They reported that before weaning (P21), rippleassociated activity represents stationary locations in space, and does not start to represent bona fide trajectories until theta sequences progressively emerge between P17 and P32.

Finally, over the last 15 years, an ever growing number of studies have addressed the network substrates of memory using causal approaches. However, these studies have generally failed to consider the temporal dimension of neuronal activity, such as manifested in cell assemblies and sequences. Experimental exploration of engrams (Semon, 1921) has benefited from the development of optogenetics (Boyden et al., 2005) and gene expression control techniques combined with immediate early gene (IEG) expression signals, to artificially manipulate memory traces (e.g., Reijmers et al., 2007; Liu et al., 2012; Cowansage et al., 2014; Redondo et al., 2014; Trouche et al., 2016). These strategies allow tagging and subsequent manipulation of neuronal populations activated during learning (for recent reviews, see Silva et al., 2009; Tonegawa et al., 2015; Guan et al., 2016; Kim et al., 2016; Poo et al., 2016). Thus, selective activation or inhibition of tagged neuronal ensembles have been shown to induce (Liu et al., 2012; Cowansage et al., 2014; Kim et al., 2014; Redondo et al., 2014) or block (Han et al., 2009; Trouche et al., 2016) the expression of memory, respectively.

One important issue with most of these studies is that they artificially activate neurons in a hyper-synchronous manner, and neglect any fine-timescale temporal information embedded in endogenous spiking patterns. In addition, because the time window for genetic labeling of neuronal networks ranges from several hours to days, the tagged population is likely to include both memory-specific neurons as well as numerous irrelevant neurons that were activated by unrelated events during the relatively long labeling time window. In any case, it remains unclear whether the tagged neurons represent a memory trace or constitute relays toward the relevant ensembles. For example, memory recall could actually result from downstream circuit computations rather than from the experimental activation of the tagged neurons per se. This would potentially restore the temporal patterns obliterated by the synchronous experimental stimulation. A parallel can be made with fRMI studies showing robust and consistent activation patterns in response to similar stimuli, despite the low temporal and spatial resolution of the technique (Guest and Love, 2017). Thus, while certain types of computation can still be detected or elicited even without having access to the fine time scale of neuronal computation, it is uncertain that they reflect actual brain computation during learning and memory. Further experimental and computational work will undoubtedly shed light on the complex issue of the temporality of circuit computations underlying memory.

\section{HIPPOCAMPAL SEQUENCES DURING EXPLORATION: DISTINCT AND COMPLEMENTARY FUNCTIONS}

\subsection{Awake Replay During Sharp-Wave Ripples}

Theta sequences are not the sole pattern of sequential activity in the awake hippocampus. While offline replay of neuronal sequences was first documented during sharp-wave ripples (SPW-Rs) of slow wave sleep (Pavlides and Winson, 1989; Wilson and McNaughton, 1994; Lee and Wilson, 2002), replay also occurs during SPW-Rs of quiet awake states, such as when the animal pauses in an environment (Figure 7; Buzsáki, 1989; Foster and Wilson, 2006; Jackson et al., 2006; O’Neill et al., 2006; Csicsvari et al., 2007; Diba and Buzsáki, 2007; Dupret et al., 2010). Foster and Wilson (2006) recorded CA1 pyramidal cell activity while rats ran back and forth between reward sites, and showed that compared to the location of their firing fields on the track, place cells tended to reactivate in reverse order during awake replay, contrary to replay during slow wave sleep (although more recent studies have since documented overlooked reverse replay during sleep as well; see e.g., Wikenheiser and Redish, 2013; Silva et al., 2015; Grosmark and Buzsáki, 2016; Ólafsdóttir et al., 2016; O’Neill et al., 2017; Drieu et al., 2018). Replay events were more readily observable on a new track, where they occurred even after the very first lap (Foster and Wilson, 2006). In addition, Diba and Buzsáki (2007) reported that replay tended to occur 


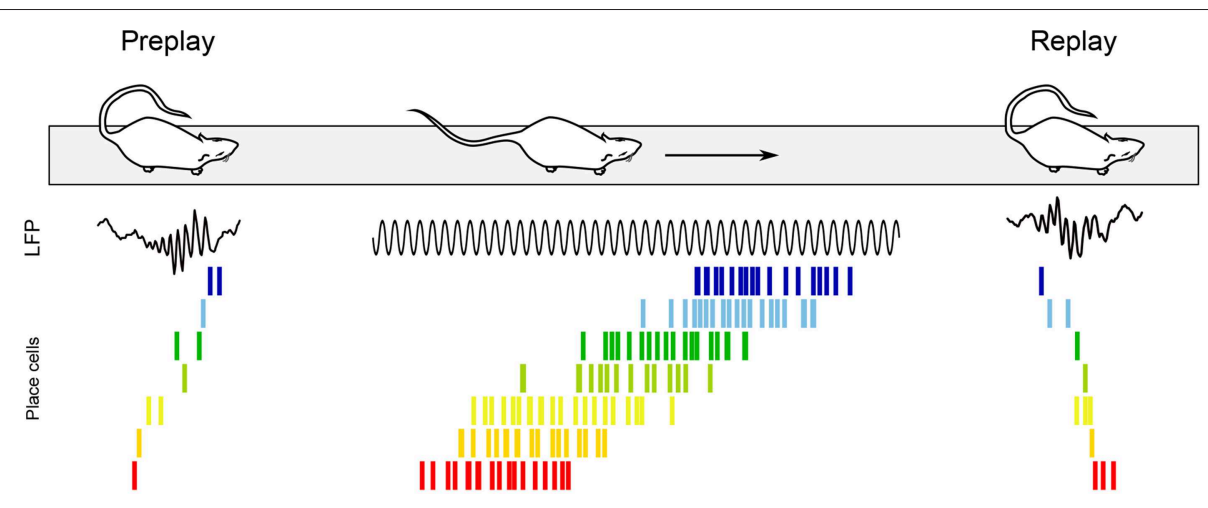

FIGURE 7 | Awake replay. Place cell sequences experienced during behavior are generated in both the forward and reverse direction during awake SPW-Rs (Diba and Buzsáki, 2007). Spike trains of place cells on the track are shown before, during and after a single traversal. Sequences that occur during track running are reactivated during SPW-Rs both before and after the run, when the rat remains immobile. Forward preplay (Left) tends to occur as the animal is getting ready to run on the track, while reverse replay (Right) tends to occur shortly after run. Black traces, CA1 local field potential.

in the reverse order upon arrival at the reward site, but in the forward order ("preplay") when rats then faced the track in anticipation of the next run (Figure 7). Interestingly, the authors documented a strong correlation between the temporal offsets of neuron pairs during forward events (preplay) and during theta oscillations when the animals were running between reward sites. In contrast, theta sequences and reverse replay events were less strongly correlated, suggesting a selective link between offline preplay and neural activity during run. The balance between replay and preplay may also depend on task contingencies, as Ólafsdóttir et al. (2017) found a greater proportion of forward replay immediately $(<5 \mathrm{~s})$ before and after movement or during short stops ( $<10 \mathrm{~s}$ ), but no directional bias during longer periods of immobility (although one cannot exclude that the apparent inconsistency with the results of Diba and Buzsáki (2007) may stem from the limited number of replay events examined in the Ólafsdóttir et al. (2017) study).

Foster and Wilson (2006) hypothesized that reverse replay could be associated with a slowly decaying dopamine signal, effectively storing a value gradient path that the animal could follow during subsequent goal-finding behavior. Conversely, forward preplay has been hypothesized to be involved in goaldirected planning (Diba and Buzsáki, 2007). Accordingly, awake replay can be influenced by reward locations (Foster and Wilson, 2006; Singer and Frank, 2009; Ambrose et al., 2016), contributes to mapping novel environments (O’Neill et al., 2006; Cheng and Frank, 2008; Roux et al., 2017), helps establishing goal-related cell assemblies (Dupret et al., 2010; Csicsvari and Dupret, 2014), supports decision making (evaluation of choices, prospection and planning) (Pfeiffer and Foster, 2013; Singer et al., 2013; Yu and Frank, 2015), and plays a role in fear (Wu et al., 2017) and episodic-like (Takahashi, 2015) memory retrieval. In addition, awake replay can span multiple SPW-R events to represent extended trajectories (Davidson et al., 2009) and link familiar paths into novel trajectories never experienced before (Gupta et al., 2010). Finally, selective suppression of awake SPWRs during a spatial alternation task fails to impair memory consolidation, and instead appears to alter working memory and decision making (Jadhav et al., 2012). In summary, awake replay has been proposed to mediate both spatial and non-spatial functions (see O’Neill et al., 2010; Buhry et al., 2011; Carr et al., 2011; and for a recent review Roumis and Frank, 2015).

Because both forward and reverse replay have a tendency to start at the current location of the animal, awake replay has been proposed to be biased by a residual, place-selective spatial tuning drive (Foster and Wilson, 2006; Csicsvari et al., 2007; Diba and Buzsáki, 2007). However, awake replay can also be modulated by non-local cues such as rewards (Pfeiffer and Foster, 2013; Singer et al., 2013) and contextual memories (Takahashi, 2015; Wu et al., 2017), or even be initiated at remote locations (Davidson et al., 2009; Karlsson and Frank, 2009; Gupta et al., 2010). This is at odds with the seminal model which posits that replay (in particular, reverse replay) can be accounted for by decreasing excitability over time (Buzsáki, 1989; Foster and Wilson, 2006). Instead, it has been proposed that awake replay could emerge from auto-associative properties of the CA3 network, in which the activation of a single cell can initiate the reactivation of an entire trajectory sequence due to recurrent connectivity established or reinforced during theta-state exploration (Lee and Wilson, 2002; O'Neill et al., 2008; Dupret et al., 2010; Silva et al., 2015), similar to the classical model of sleep replay. In turn however, this model cannot account for the (weak) link between the time spent exploring different areas of the environment and the propensity of replay to represent the corresponding trajectories (Gupta et al., 2010; but see O'Neill et al., 2008), the construction of neverexperienced sequences (Gupta et al., 2010), nor the differential balance of forward and backward replay across behavioral states (Wikenheiser and Redish, 2013). It is therefore likely that replay results from a combination of these mutually non-exclusive mechanisms. Hence, Atherton et al. (2015) have suggested that the lingering excitability model largely dictates local replaying sequences during awake behavior, while the synaptic plasticity model contributes to subsequent nonlocal awake replay (and 
sleep replay). Finally, neuromodulatory activity may also exert an important role (Atherton et al., 2015): dopamine release during novel maze exploration and reward-driven spatial tasks may promote the strengthening of new place cell assemblies and place-reward associations (Benchenane et al., 2010; Dupret et al., 2010; McNamara et al., 2014; de Lavilléon et al., 2015; Ambrose et al., 2016; Takahashi et al., 2016; Takeuchi et al., 2016), while cholinergic tone may influence the direction of replay in different behavioral states (Marrosu et al., 1995; Csicsvari et al., 2007; Wikenheiser and Redish, 2013; Saravanan et al., 2015).

\subsection{Functional Dissociation Between Theta Sequences and Awake Replay?}

The above discussion suggests a functional dissociation between theta sequences and awake replay, where theta sequences would store initial memory traces about ongoing experiences as well as recall previously formed memories and enhance them to include goal-directed information, while awake replay would play an instrumental role in learning goal locations and planning upcoming trajectories. Complementing these mechanisms, sleep replay would sustain memory consolidation. As it turns out, a closer examination of the available data suggests a more complex picture. Below, we point out some of the current inconsistencies and overlooked findings.

\subsubsection{Memory Maintenance and Retrieval}

Wang et al. (2016) inactivated the medial septum in rats trained on a delayed spatial alternation task. They found that theta sequences were disrupted and performance was impaired. Surprisingly, awake replay was preserved. The authors concluded that theta sequences on the maze (and in the running wheel during delays), but not awake replay, are required to recall episodic memories even in well-trained animals. Alternatively, awake replay would be required only for initial learning of a task but not once the environment and the task have become familiar. This would be consistent with the finding that suppression of awake SPW-Rs during the first 8 days of learning impairs working memory in a spatial alternation task (Jadhav et al., 2012). Yet another possible explanation for the apparently predominant role of theta sequences over awake replay may be that the rats spent the delay period running in the wheel, which may have favored neuronal events occurring in the theta state. In any case, these results still argue that theta sequences and awake replay do not play equivalent, interchangeable roles.

Wu et al. (2017) trained animals in a foot-shock avoidance task and reported that avoidance of the shock zone was concomitant with awake replay, but not theta sequences, of paths leading from current position to the foot-shock zone. This further supports distinct functional roles for theta sequences and awake replay in long-term contextual memory retrieval.

\subsubsection{Planning and Decision-Making}

Another notable difference between theta sequences and awake replay is that theta sequences seem to be restricted to short distances always encompassing current location, occasionally extending to further locations (Johnson et al., 2007; Wikenheiser and Redish, 2015b), whereas awake replay can span much longer distances (up to $\geq 10 \mathrm{~m}$; Davidson et al., 2009) and be decoupled from current experience (Karlsson and Frank, 2009; Gupta et al., 2010). Therefore, while theta sequences may be relevant for spatial memory, in particular to allow trajectory encoding and retrieval (Hasselmo and Eichenbaum, 2005; Dragoi and Buzsáki, 2006; Foster and Wilson, 2007; Robbe and Buzsáki, 2009; Wang et al., 2015; Drieu et al., 2018), awake replay may be necessary to associate paths and events disconnected in time and space, allowing flexibility and adaptive behaviors (Gupta et al., 2010).

During deliberative behavior at a choice point (Muenzinger and Gentry, 1931; Redish, 2016), theta sequences reflect possible paths (Johnson et al., 2007). Papale et al. (2016) reported that the occurrence rate of VTE at the choice point was inversely correlated to that of SPW-Rs at the reward sites on the same lap. Further, the SPW-R rate on a given lap inversely correlated with the likelihood of subsequent VTE events. Finally, interruption of SPW-Rs during learning led to an increased prevalence of VTE. This suggests that SPW-Rs are involved in ongoing consolidation, while VTE sequences participate in a decisionmaking process. On the other hand, SPW-Rs are also known to occur at decision points during learning (Jadhav et al., 2012), which could reflect an implication in decision-making processes, blurring the proposed functional distinction between theta sequences and awake replay. Disentangling these matters is further hindered by the fact that ripple disruption in Jadhav et al. (2012) was not triggered with spatial specificity: ripples were disrupted irrespective of the location of the rat. Therefore, the specific functional contribution of ripples occurring at decision points remains to be elucidated.

\subsubsection{Learning and Consolidation}

Both theta and SPW-Rs are propitious to synaptic potentiation between sequentially activated cell assemblies (Skaggs et al., 1996; Magee and Johnston, 1997; Markram et al., 1997; Dragoi et al., 2003; Wójtowicz and Mozrzymas, 2015; Sadowski et al., 2016; Cobar et al., 2017; Drieu et al., 2018). Therefore, in principle neuronal sequences in both states could lead to learning and reinforcement of sequential activity.

In the machine learning literature, reverse replay has been repeatedly reported to enhance reinforcement learning, in particular temporal difference learning (Cazé et al., 2018; Mattar and Daw, 2018). Interestingly, awake replay during brief pauses in exploration and at reward sites largely occur in reverse order (O’Neill et al., 2008; Wikenheiser and Redish, 2013), identifying a potential mechanisms to support learning of trajectories and goal locations (Singer et al., 2013). In addition, working memory deficits have been observed following suppression of SPW-Rs that occurred mainly at reward locations (Jadhav et al., 2012). Theta sequences on the other hand mainly reflect currently experienced trajectories in the forward order. Yet, a small fraction of theta cycles do contain significant reverse theta sequences (Gupta et al., 2012; Zheng et al., 2016; our own unpublished observations). The function of reverse theta sequences remains unclear. Similarly, although this has been overlooked in initial descriptions of sleep replay, recent studies have reported abundant reverse replay during sleep as well (Wikenheiser and Redish, 2013; Silva et al., 2015; Grosmark 
and Buzsáki, 2016; Ólafsdóttir et al., 2016; O’Neill et al., 2017; Drieu et al., 2018). Again, these considerations dim the usual theoretical distinction between (forward) theta sequences for memory encoding, (reverse) awake replay for goal learning, and (forward) sleep replay for memory consolidation.

An additional complexity is that SPW-R incidence and replay strength and accuracy vary according to the length, shape and complexity of the maze, as well as the cognitive demand of the task (Jackson et al., 2006; Dupret et al., 2010; Pfeiffer and Foster, 2013; Singer et al., 2013). Increases in both SPW$\mathrm{R}$ occurrence rate and replay strength can even be observed in familiar environments (Jackson et al., 2006; Dupret et al., 2010; Pfeiffer and Foster, 2013; Singer et al., 2013), perhaps because awake replay helps stabilize hippocampal place fields when new configurations are learned within a familiar map (Roux et al., 2017).

\section{CONCLUSION}

Sequences of hippocampal cell assemblies formed during individual theta cycles represent the time-compressed ongoing trajectory of the animal. These theta sequences are thought to emerge from ensembles of phase precessing cells, although it is currently unclear whether this requires additional coordination between participating neurons. Numerous computational models have proposed cellular and network mechanisms to account for theta sequences, but the precise neurophysiological mechanisms of phase precession remain elusive. While initial reports emphasized the presence of sequential activity during single theta cycles, recent studies have increasingly recognized that a notable fraction of theta cycles contain noncanonical activity, including seemingly random noise and even reverse sequences.

\section{REFERENCES}

Aghajan, Z. M., Acharya, L., Moore, J. J., Cushman, J. D., Vuong, C., and Mehta, M. R. (2015). Impaired spatial selectivity and intact phase precession in twodimensional virtual reality. Nat. Neurosci. 18, 121-128. doi: 10.1038/nn.3884

Alonso, A., and Köhler, C. (1982). Evidence for separate projections of hippocampal pyramidal and non-pyramidal neurons to different parts of the septum in the rat brain. Neurosci. Lett. 31, 209-214. doi: 10.1016/0304-3940(82)90021-0

Ambrose, R. E., Pfeiffer, B. E., and Foster, D. J. (2016). Reverse replay of hippocampal place cells is uniquely modulated by changing reward. Neuron 91 , 1124-1136. doi: 10.1016/j.neuron.2016.07.047

Amemiya, S., and Redish, A. D. (2016). Manipulating decisiveness in decision making: Effects of clonidine on hippocampal search strategies. J. Neurosci. 36, 814-827. doi: 10.1523/JNEUROSCI.2595-15.2016

Andersen, P., Morris, R., Amaral, D., Bliss, T., and O'Keefe, J. (2007). The Hippocampus Book. New York, NY: Oxford University Press.

Aronov, D., Nevers, R., and Tank, D. W. (2017). Mapping of a non-spatial dimension by the hippocampal-entorhinal circuit. Nature 543, 719-722. doi: 10.1038/nature21692

Atherton, L. A., Dupret, D., and Mellor, J. R. (2015). Memory trace replay: the shaping of memory consolidation by neuromodulation. Trends Neurosci. 38, 560-570. doi: 10.1016/j.tins.2015.07.004

Battaglia, F. P., Sutherland, G. R., and McNaughton, B. L. (2004). Local sensory cues and place cell directionality: additional evidence of
Theta sequences chunk information into time windows conducive to classical synaptic plasticity mechanisms, and appear to support the initial storage of memory traces. Within familiar environments, theta sequences allow the maintenance (and possibly the update) of planned trajectories in working memory. During VTE, extended neuronal sweeps may support deliberative processes. Awake replay could also support trajectory planning, on-line consolidation and reconsolidation, as well as on-line retrieval of long-term memory. Thus, it remains currently unclear which specific functions are sustained by theta sequences, awake replay, or both.

Finally, a growing number of studies have provided evidence that hippocampal activity can also convey non-spatial information, including about objects, odors, sound sequences, time, goals, fear, etc. (e.g., Wood et al., 1999; Moita et al., 2003; Macdonald et al., 2011; Aronov et al., 2017). This suggests a general mechanism for encoding continuous, task-relevant variables. Recent findings indicate that phase precession may also represent a potential code for non-spatial features (Terada et al., 2017). Future work will be required to determine how non-spatial information is integrated at the fine timescale during hippocampal computation.

\section{AUTHOR CONTRIBUTIONS}

All authors listed have made a substantial, direct and intellectual contribution to the work, and approved it for publication.

\section{FUNDING}

This work was supported by the Agence Nationale de la Recherche (ANR-15-CE16-0001-02) and Collège de France (CD).

prospective coding in the hippocampus. J. Neurosci. 24, 4541-4550. doi: 10.1523/JNEUROSCI.4896-03.2004

Belluscio, M. A., Mizuseki, K., Schmidt, R., Kempter, R., and Buzsáki, G. (2012). Cross-frequency phase-phase coupling between theta and gamma oscillations in the hippocampus. J. Neurosci. 32, 423-435. doi: 10.1523/JNEUROSCI.4122-11.2012

Benchenane, K., Peyrache, A., Khamassi, M., Tierney, P. L., Gioanni, Y., Battaglia, F. P., et al. (2010). Coherent theta oscillations and reorganization of spike timing in the hippocampal- prefrontal network upon learning. Neuron 66, 921-936. doi: 10.1016/j.neuron.2010.05.013

Bender, F., Gorbati, M., Cadavieco, M. C., Denisova, N., Gao, X., Holman, C., et al. (2015). Theta oscillations regulate the speed of locomotion via a hippocampus to lateral septum pathway. Nat. Commun. 6:8521. doi: 10.1038/ncomms9521

Bieri, K. W., Bobbitt, K. N., and Colgin, L. L. (2014). Slow and fast $\gamma$ rhythms coordinate different spatial coding modes in hippocampal place cells. Neuron 82, 670-681. doi: 10.1016/j.neuron.2014.03.013

Bittner, K. C., Milstein, A. D., Grienberger, C., Romani, S., and Magee, J. C. (2017). Behavioral time scale synaptic plasticity underlies cal place fields. Science 357, 1033-1036. doi: 10.1126/science.aan3846

Bland, B. H., Bland, C. E., and MacIver, M. B. (2016). Median raphe stimulationinduced motor inhibition concurrent with suppression of type 1 and type 2 hippocampal theta. Hippocampus 26, 289-300. doi: 10.1002/hipo.22521

Bose, A., and Recce, M. (2001). Phase precession and phase-locking of hippocampal pyramidal cells. Hippocampus 11, 204-215. doi: 10.1002/hipo.1038 
Boyden, E. S., Zhang, F., Bamberg, E., Nagel, G., and Deisseroth, K. (2005). Millisecond-timescale, genetically targeted optical control of neural activity. Nat. Neurosci. 8, 1263-1268. doi: 10.1038/nn1525

Bragin, A., Jandó, G., Nádasdy, Z., Hetke, J., Wise, K., and Buzsáki, G. (1995). Gamma (40-100 hz) oscillation in the hippocampus of the behaving rat. J. Neurosci. 15(1 Pt 1):47-60. doi: 10.1523/JNEUROSCI.15-01-00047.1995

Buhry, L., Azizi, A. H., and Cheng, S. (2011). Reactivation, replay, and preplay: how it might all fit together. Neural Plast. 2011:203462. doi: 10.1155/2011/203462

Burgess, N., Barry, C., and O'Keefe, J. (2007). An oscillatory interference model of grid cell firing. Hippocampus 17, 801-812. doi: 10.1002/hipo.20327

Burgess, N., and O'Keefe, J. (2011). Models of place and grid cell firing and theta rhythmicity. Curr. Opin. Neurobiol. 21, 734-744. doi: 10.1016/j.conb.2011.07.002

Burgess, N., Recce, M., and O'Keefe, J. (1994). A model of hippocampal function. Neural Netw. 7, 1065-1081. doi: 10.1016/S0893-6080(05)80159-5

Buzsáki, G. (1989). Two-stage model of memory trace formation: a role for "noisy" brain states. Neuroscience 31, 551-570. doi: 10.1016/0306-4522(89)90423-5

Buzsáki, G. (2010). Neural syntax: cell assemblies, synapsembles, and readers. Neuron 68, 362-385. doi: 10.1016/j.neuron.2010.09.023

Buzsáki, G., Csicsvari, J., Dragoi, G., Harris, K., Henze, D., and Hirase, H. (2002). Homeostatic maintenance of neuronal excitability by burst discharges in vivo. Cereb. Cortex 12, 893-899. doi: 10.1093/cercor/12.9.893

Buzsáki, G., Czopf, J., Kondákor, I., and Kellényi, L. (1986). Laminar distribution of hippocampal rhythmic slow activity (rsa) in the behaving rat: current-source density analysis, effects of urethane and atropine. Brain Res. 365, 125-137. doi: 10.1016/0006-8993(86)90729-8

Buzsáki, G., and Draguhn, A. (2004). Neuronal oscillations in cortical networks. Science 304, 1926-1929. doi: 10.1126/science. 1099745

Buzsáki, G., Horváth, Z., Urioste, R., Hetke, J., and Wise, K. (1992). Highfrequency network oscillation in the hippocampus. Science 256, 1025-1027. doi: 10.1126/science. 1589772

Buzsáki, G., Leung, L. W., and Vanderwolf, C. H. (1983). Cellular bases of hippocampal eeg in the behaving rat. Brain Res. 287, 139-171. doi: 10.1016/0165-0173(83)90037-1

Buzsáki, G., and Moser, E. I. (2013). Memory, navigation and theta rhythm in the hippocampal-entorhinal system. Nat. Neurosci. 16, 130-138. doi: 10.1038/nn.3304

Buzsáki, G., and Schomburg, E. W. (2015). What does gamma coherence tell us about inter-regional neural communication? Nat. Neurosci. 18, 484-489. doi: $10.1038 / \mathrm{nn} .3952$

Cabral, H. O., Vinck, M., Fouquet, C., Pennartz, C. M. A., Rondi-Reig, L., and Battaglia, F. P. (2014). Oscillatory dynamics and place field maps reflect hippocampal ensemble processing of sequence and place memory under nmda receptor control. Neuron 81, 402-415. doi: 10.1016/j.neuron.2013. 11.010

Carr, M. F., Jadhav, S. P., and Frank, L. M. (2011). Hippocampal replay in the awake state: a potential substrate for memory consolidation and retrieval. Nat. Neurosci. 14, 147-153. doi: 10.1038/nn.2732

Cazé, R., Khamassi, M., Aubin, L., and Girard, B. (2018). Hippocampal replays under the scrutiny of reinforcement learning models. J. Neurophysiol. 120, 2877-2896. doi: 10.1152/jn.00145.2018

Cei, A., Girardeau, G., Drieu, C., Kanbi, K. E., and Zugaro, M. (2014). Reversed theta sequences of hippocampal cell assemblies during backward travel. Nat. Neurosci. 17, 719-724. doi: 10.1038/nn.3698

Chadwick, A., van Rossum, M. C., and Nolan, M. F. (2016). Flexible theta sequence compression mediated via phase precessing interneurons. Elife 5:e20349. doi: 10.7554/eLife.20349

Chadwick, A., van Rossum, M. C. W., and Nolan, M. F. (2015). Independent theta phase coding accounts for cal population sequences and enables flexible remapping. ELife 4:e03542. doi: 10.7554/eLife.03542

Chance, F. S. (2012). Hippocampal phase precession from dual input components. J. Neurosci. 32, 16693-16703. doi: 10.1523/JNEUROSCI.2786-12.2012

Chen, G., King, J. A., Burgess, N., and O'Keefe, J. (2013). How vision and movement combine in the hippocampal place code. Proc. Natl. Acad. Sci. U.S.A. 110, 378-383. doi: 10.1073/pnas.1215834110

Cheng, S., and Frank, L. M. (2008). New experiences enhance coordinated neural activity in the hippocampus. Neuron 57, 303-313. doi: 10.1016/j.neuron.2007.11.035
Climer, J. R., Newman, E. L., and Hasselmo, M. E. (2013). Phase coding by grid cells in unconstrained environments: two-dimensional phase precession. Eur. J. Neurosci. 38, 2526-2541. doi: 10.1111/ejn.12256

Cobar, L. F., Yuan, L., and Tashiro, A. (2017). Place cells and long-term potentiation in the hippocampus. Neurobiol. Learn. Mem. 138, 206-214. doi: 10.1016/j.nlm.2016.10.010

Colgin, L. L., Denninger, T., Fyhn, M., Hafting, T., Bonnevie, T., Jensen, O., et al. (2009). Frequency of gamma oscillations routes flow of information in the hippocampus. Nature 462, 353-357. doi: 10.1038/nature08573

Cowansage, K. K., Shuman, T., Dillingham, B. C., Chang, A., Golshani, P., and Mayford, M. (2014). Direct reactivation of a coherent neocortical memory of context. Neuron 84, 432-41. doi: 10.1016/j.neuron.2014.09.022

Csicsvari, J., and Dupret, D. (2014). Sharp wave/ripple network oscillations and learning-associated hippocampal maps. Philos. Trans. R. Soc. Lond. B Biol. Sci. 369:20120528. doi: 10.1098/rstb.2012.0528

Csicsvari, J., Henze, D. A., Jamieson, B., Harris, K. D., Sirota, A., Barthó, P., et al. (2003). Massively parallel recording of unit and local field potentials with silicon-based electrodes. J. Neurophysiol. 90, 1314-1323. doi: $10.1152 /$ jn.00116.2003

Csicsvari, J., Hirase, H., Czurkó, A., Mamiya, A., and Buzsáki, G. (1999). Fast network oscillations in the hippocampal cal region of the behaving rat. $J$. Neurosci. 19:RC20. doi: 10.1523/JNEUROSCI.19-16-j0001.1999

Csicsvari, J., O'Neill, J., Allen, K., and Senior, T. (2007). Place-selective firing contributes to the reverse-order reactivation of cal pyramidal cells during sharp waves in open-field exploration. Eur. J. Neurosci. 26, 704-716. doi: 10.1111/j.1460-9568.2007.05684.x

Cutsuridis, V., and Hasselmo, M. (2012). Gabaergic contributions to gating, timing, and phase precession of hippocampal neuronal activity during theta oscillations. Hippocampus 22, 1597-1621. doi: 10.1002/hipo.21002

Czurkó, A., Hirase, H., Csicsvari, J., and Buzsáki, G. (1999). Sustained activation of hippocampal pyramidal cells by 'space clamping' in a running wheel. Eur. J. Neurosci. 11, 344-352. doi: 10.1046/j.1460-9568.1999.00446.x

Davidson, T. J., Kloosterman, F., and Wilson, M. A. (2009). Hippocampal replay of extended experience. Neuron 63, 497-507. doi: 10.1016/j.neuron.2009.07.027

de Lavilléon, G., Lacroix, M. M., Rondi-Reig, L., and Benchenane, K. (2015). Explicit memory creation during sleep demonstrates a causal role of place cells in navigation. Nat. Neurosci. 18, 493-495. doi: 10.1038/ nn.3970

Deshmukh, S. S., and Knierim, J. J. (2011). Representation of non-spatial and spatial information in the lateral entorhinal cortex. Front. Behav. Neurosci. 5:69. doi: 10.3389/fnbeh.2011.00069

Diba, K., and Buzsáki, G. (2007). Forward and reverse hippocampal place-cell sequences during ripples. Nat. Neurosci. 10, 1241-1242. doi: 10.1038/nn1961

Domnisoru, C., Kinkhabwala, A. A., and Tank, D. W. (2013). Membrane potential dynamics of grid cells. Nature 495, 199-204. doi: 10.1038/nature11973

Dragoi, G., and Buzsáki, G. (2006). Temporal encoding of place sequences by hippocampal cell assemblies. Neuron 50, 145-157. doi: 10.1016/j.neuron.2006.02.023

Dragoi, G., Harris, K. D., and Buzsáki, G. (2003). Place representation within hippocampal networks is modified by long-term potentiation. Neuron 39, 843-853. doi: 10.1016/S0896-6273(03)00465-3

Dragoi, G., and Tonegawa, S. (2011). Preplay of future place cell sequences by hippocampal cellular assemblies. Nature 469, 397-401. doi: $10.1038 /$ nature09633

Dragoi, G., and Tonegawa, S. (2013). Distinct preplay of multiple novel spatial experiences in the rat. Proc. Natl. Acad. Sci. U.S.A. 110, 9100-9105. doi: 10.1073/pnas.1306031110

Drieu, C., Todorova, R., and Zugaro, M. (2018). Nested sequences of hippocampal assemblies during behavior support subsequent sleep replay. Science 362, 675679. doi: 10.1126/science.aat 2952

Dupret, D., O'Neill, J., and Csicsvari, J. (2013). Dynamic reconfiguration of hippocampal interneuron circuits during spatial learning. Neuron 78, 166-180. doi: 10.1016/j.neuron.2013.01.033

Dupret, D., O'Neill, J., Pleydell-Bouverie, B., and Csicsvari, J. (2010). The reorganization and reactivation of hippocampal maps predict spatial memory performance. Nat. Neurosci. 13, 995-1002. doi: 10.1038/nn.2599

Ebbesen, C. L., Reifenstein, E. T., Tang, Q., Burgalossi, A., Ray, S., Schreiber, S., et al. (2016). Cell type-specific differences in spike timing and spike shape in 
the rat parasubiculum and superficial medial entorhinal cortex. Cell Rep. 16, 1005-1015. doi: 10.1016/j.celrep.2016.06.057

Ego-Stengel, V., and Wilson, M. A. (2007). Spatial selectivity and theta phase precession in cal interneurons. Hippocampus 17, 161-174. doi: 10.1002/hipo.20253

Ego-Stengel, V., and Wilson, M. A. (2010). Disruption of ripple-associated hippocampal activity during rest impairs spatial learning in the rat. Hippocampus 20, 1-10. doi: 10.1002/hipo.20707

Eichenbaum, H. (2017). The role of the hippocampus in navigation is memory. J. Neurophysiol. 117, 1785-1796. doi: 10.1152/jn.00005.2017

Epsztein, J., Brecht, M., and Lee, A. K. (2011). Intracellular determinants of hippocampal cal place and silent cell activity in a novel environment. Neuron 70, 109-120. doi: 10.1016/j.neuron.2011.03.006

Evstratova, A., Chamberland, S., and Topolnik, L. (2011). Cell type-specific and activity-dependent dynamics of action potential-evoked ca2+ signals in dendrites of hippocampal inhibitory interneurons. J. Physiol. (Lond.) 589(Pt 8):1957-1977. doi: 10.1113/jphysiol.2010.204255

Feng, T., Silva, D., and Foster, D. J. (2015). Dissociation between the experiencedependent development of hippocampal theta sequences and single-trial phase precession. J. Neurosci. 35, 4890-4902. doi: 10.1523/JNEUROSCI.261414.2015

Ferguson, K. A., Huh, C. Y. L., Amilhon, B., Manseau, F., Williams, S., and Skinner, F. K. (2015). Network models provide insights into how orienslacunosum-moleculare and bistratified cell interactions influence the power of local hippocampal cal theta oscillations. Front. Syst. Neurosci. 9:110. doi: 10.3389/fnsys.2015.00110

Fernández-Ruiz, A., Oliva, A., Nagy, G. A., Maurer, A. P., Berényi, A., and Buzsáki, G. (2017). Entorhinal-ca3 dual-input control of spike timing in the hippocampus by theta-gamma coupling. Neuron 93, 1213-1226-e5. doi: 10.1016/j.neuron.2017.02.017

Fisher, R. S., and Blum, D. (1999). Epilepsy surgery where there is dual pathology. Lancet 354, 267-268. doi: 10.1016/S0140-6736(99)00180-4

Foster, D. J., and Knierim, J. J. (2012). Sequence learning and the role of the hippocampus in rodent navigation. Curr. Opin. Neurobiol. 22, 294-300. doi: 10.1016/j.conb.2011.12.005

Foster, D. J., and Wilson, M. A. (2006). Reverse replay of behavioural sequences in hippocampal place cells during the awake state. Nature 440, 680-683. doi: 10.1038/nature04587

Foster, D. J., and Wilson, M. A. (2007). Hippocampal theta sequences. Hippocampus 17, 1093-1099. doi: 10.1002/hipo.20345

Foster, T. C., Castro, C. A., and McNaughton, B. L. (1989). Spatial selectivity of rat hippocampal neurons: dependence on preparedness for movement. Science 244, 1580-1582. doi: 10.1126/science.2740902

Freund, T. F., and Buzsáki, G. (1996). Interneurons of the hippocampus. Hippocampus 6, 347-470.

Geisler, C., Diba, K., Pastalkova, E., Mizuseki, K., Royer, S., and Buzsáki, G. (2010). Temporal delays among place cells determine the frequency of population theta oscillations in the hippocampus. Proc. Natl. Acad. Sci. U.S.A. 107, 7957-7962. doi: 10.1073/pnas.0912478107

Geisler, C., Robbe, D., Zugaro, M., Sirota, A., and Buzsáki, G. (2007). Hippocampal place cell assemblies are speed-controlled oscillators. Proc. Natl. Acad. Sci. U.S.A. 104, 8149-8154. doi: 10.1073/pnas.0610121104

Gilbert, P. E., and Brushfield, A. M. (2009). The role of the ca3 hippocampal subregion in spatial memory: a process oriented behavioral assessment. Prog. Neuropsychopharmacol. Biol. Psychiatry 33, 774-781. doi: 10.1016/j.pnpbp.2009.03.037

Girardeau, G., Benchenane, K., Wiener, S. I., Buzsáki, G., and Zugaro, M. B. (2009). Selective suppression of hippocampal ripples impairs spatial memory. Nat. Neurosci. 12, 1222-1223. doi: 10.1038/nn.2384

Goutagny, R., Jackson, J., and Williams, S. (2009). Self-generated theta oscillations in the hippocampus. Nat. Neurosci. 12, 1491-1493. doi: 10.1038/nn.2440

Grastyán, E., Karmos, G., Vereczkey, L., Martin, J., and Kellenyi, L. (1965). Hypothalamic motivational processes as reflected by their hippocampal electrical correlates. Science 149, 91-93. doi: 10.1126/science.149.3679.91

Grienberger, C., Milstein, A. D., Bittner, K. C., Romani, S., and Magee, J. C. (2017). Inhibitory suppression of heterogeneously tuned excitation enhances spatial coding in cal place cells. Nat. Neurosci. 20, 417-426. doi: 10.1038/ nn. 4486
Grosmark, A. D., and Buzsáki, G. (2016). Diversity in neural firing dynamics supports both rigid and learned hippocampal sequences. Science 351, 14401443. doi: $10.1126 /$ science.aad 1935

Guan, J. S., Jiang, J., Xie, H., and Liu, K. Y. (2016). How does the sparse memory "engram" neurons encode the memory of a spatial-temporal event? Front. Neural Circuits 10:61. doi: 10.3389/fncir.2016.00061

Guest, O., and Love, B. C. (2017). What the success of brain imaging implies about the neural code. Elife 6:e21397. doi: 10.7554/eLife.21397

Gupta, A. S., van der Meer, M. A. A., Touretzky, D. S., and Redish, A. D. (2010). Hippocampal replay is not a simple function of experience. Neuron 65, 695-705. doi: 10.1016/j.neuron.2010.01.034

Gupta, A. S., van der Meer, M. A. A., Touretzky, D. S., and Redish, A. D. (2012). Segmentation of spatial experience by hippocampal $\theta$ sequences. Nat. Neurosci. 15, 1032-1039. doi: 10.1038/nn.3138

Hafting, T., Fyhn, M., Bonnevie, T., Moser, M.-B., and Moser, E. I. (2008). Hippocampus-independent phase precession in entorhinal grid cells. Nature 453, 1248-1252. doi: 10.1038/nature06957

Han, J. H., Kushner, S. A., Yiu, A. P., Hsiang, H. L. L., Buch, T., Waisman, A., et al. (2009). Selective erasure of a fear memory. Science 323, 1492-1496. doi: 10.1126/science.1164139

Hansen, A. K., Nedergaard, S., and Andreasen, M. (2014). Intrinsic ca2+dependent theta oscillations in apical dendrites of hippocampal cal pyramidal cells in vitro. J. Neurophysiol. 112, 631-643. doi: 10.1152/jn.00753.2013

Harper, R. M. (1971). Frequency changes in hippocampal electrical activity during movement and tonic immobility. Physiol. Behav. 7, 55-58. doi: 10.1016/0031-9384(71)90235-6

Harris, K. D. (2005). Neural signatures of cell assembly organization. Nat. Rev. Neurosci. 6, 399-407. doi: 10.1038/nrn1669

Harris, K. D., Csicsvari, J., Hirase, H., Dragoi, G., and Buzsáki, G. (2003). Organization of cell assemblies in the hippocampus. Nature 424, 552-556. doi: $10.1038 /$ nature 01834

Harris, K. D., Henze, D. A., Hirase, H., Leinekugel, X., Dragoi, G., Czurkó, A., et al. (2002). Spike train dynamics predicts theta-related phase precession in hippocampal pyramidal cells. Nature 417, 738-741. doi: 10.1038/nature00808

Harvey, C. D., Collman, F., Dombeck, D. A., and Tank, D. W. (2009). Intracellular dynamics of hippocampal place cells during virtual navigation. Nature 461, 941-946. doi: 10.1038/nature08499

Hasselmo, M. E., Bodelón, C., and Wyble, B. P. (2002). A proposed function for hippocampal theta rhythm: separate phases of encoding and retrieval enhance reversal of prior learning. Neural Comput. 14, 793-817. doi: 10.1162/089976602317318965

Hasselmo, M. E., and Eichenbaum, H. (2005). Hippocampal mechanisms for the context-dependent retrieval of episodes. Neural Netw. 18, 1172-1190. doi: 10.1016/j.neunet.2005.08.007

Hinman, J. R., Penley, S. C., Escabí, M. A., and Chrobak, J. J. (2013). Ketamine disrupts theta synchrony across the septotemporal axis of the cal region of hippocampus. J. Neurophysiol. 109, 570-579. doi: 10.1152/jn.00561.2012

Hinman, J. R., Penley, S. C., Long, L. L., Escabí, M. A., and Chrobak, J. J. (2011). Septotemporal variation in dynamics of theta: speed and habituation. J. Neurophysiol. 105, 2675-2686. doi: 10.1152/jn.00837.2010

Hirase, H., HH7, C., Csicsvari, J., and Buzsáki, G. (1999). Firing rate and thetaphase coding by hippocampal pyramidal neurons during 'space clamping'. Eur. J. Neurosci. 11, 4373-4380. doi: 10.1046/j.1460-9568.1999.00853.x

Huxter, J., Burgess, N., and O'Keefe, J. (2003). Independent rate and temporal coding in hippocampal pyramidal cells. Nature 425, 828-832. doi: 10.1038 /nature 02058

Huxter, J. R., Senior, T. J., Allen, K., and Csicsvari, J. (2008). Theta phasespecific codes for two-dimensional position, trajectory and heading in the hippocampus. Nat. Neurosci. 11, 587-594. doi: 10.1038/nn.2106

Itskov, V., Curto, C., and Harris, K. D. (2008). Valuations for spike train prediction. Neural Computat. 20, 644-667. doi: 10.1162/neco.2007.3179

Jackson, J., Amilhon, B., Goutagny, R., Bott, J.-B., Manseau, F., Kortleven, C., et al. (2014). Reversal of theta rhythm flow through intact hippocampal circuits. Nat. Neurosci. 17, 1362-1370. doi: 10.1038/nn.3803

Jackson, J., Dickson, C. T., and Bland, B. H. (2008). Median raphe stimulation disrupts hippocampal theta via rapid inhibition and state-dependent phase reset of theta-related neural circuitry. J. Neurophysiol. 99, 3009-3026. doi: $10.1152 /$ jn.00065.2008 
Jackson, J. C., Johnson, A., and Redish, A. D. (2006). Hippocampal sharp waves and reactivation during awake states depend on repeated sequential experience. J. Neurosci. 26, 12415-12426. doi: 10.1523/JNEUROSCI.4118-06.2006

Jadhav, S. P., Kemere, C., German, P. W., and Frank, L. M. (2012). Awake hippocampal sharp-wave ripples support spatial memory. Science 336, 1454-1458. doi: 10.1126/science.1217230

Jaramillo, J., and Kempter, R. (2017). Phase precession: a neural code underlying episodic memory? Curr. Opin. Neurobiol. 43, 130-138. doi: 10.1016/j.conb.2017.02.006

Jeewajee, A., Barry, C., Douchamps, V., Manson, D., Lever, C., and Burgess, N. (2014). Theta phase precession of grid and place cell firing in open environments. Philos. Trans. R. Soc. Lond. B Biol. Sci. 369:20120532. doi: $10.1098 /$ rstb.2012.0532

Jensen, O. and Lisman, J. E. (1996). Theta/gamma networks with slow nmda channels learn sequences and encode episodic memory: role of nmda channels in recall. Learn. Mem. 3, 264-278. doi: 10.1101/lm.3.2-3.264

Jensen, O., and Lisman, J. E. (2000). Position reconstruction from an ensemble of hippocampal place cells: contribution of theta phase coding. J. Neurophysiol. 83, 2602-2609. doi: 10.1152/jn.2000.83.5.2602

Jensen, O., and Lisman, J. E. (2005). Hippocampal sequence-encoding driven by a cortical multi-item working memory buffer. Trends Neurosci. 28, 67-72. doi: 10.1016/j.tins.2004.12.001

Jezek, K., Henriksen, E. J., Treves, A., Moser, E. I., and Moser, M.-B. (2011). Theta-paced flickering between place-cell maps in the hippocampus. Nature 478, 246-249. doi: 10.1038/nature10439

Ji, D., and Wilson, M. A. (2007). Coordinated memory replay in the visual cortex and hippocampus during sleep. Nat. Neurosci. 10, 100-107. doi: $10.1038 / \mathrm{nn} 1825$

Johnson, A., van der Meer, M. A., and Redish, A. D. (2007). Integrating hippocampus and striatum in decision-making. Curr. Opin. Neurobiol. 17, 692-697. doi: 10.1016/j.conb.2008.01.003

Jones, M. W., and Wilson, M. A. (2005). Theta rhythms coordinate hippocampalprefrontal interactions in a spatial memory task. PLoS Biol. 3:e402. doi: 10.1371/journal.pbio.0030402

Jouvet, M. (1969). Biogenic amines and the states of sleep. Science 163, 32-41. doi: 10.1126/science.163.3862.32

Kamondi, A., Acsády, L., Wang, X. J., and Buzsáki, G. (1998). Theta oscillations in somata and dendrites of hippocampal pyramidal cells in vivo: activitydependent phase-precession of action potentials. Hippocampus 8, 244-261.

Karlsson, M. P., and Frank, L. M. (2009). Awake replay of remote experiences in the hippocampus. Nat. Neurosci. 12, 913-918. doi: 10.1038/nn.2344

Kiehn, O. (2016). Decoding the organization of spinal circuits that control locomotion. Nat. Rev. Neurosci. 17, 224-238. doi: 10.1038/nrn.2016.9

Kim, D., Jeong, H., Lee, J., Ghim, J. W., Her, E. S., Lee, S. H., et al. (2016). Distinct roles of parvalbumin- and somatostatin-expressing interneurons in working memory. Neuron 92, 902-915. doi: 10.1016/j.neuron.2016. 09.023

Kim, J., Kwon, J. T., Kim, H. S., Josselyn, S. A., and Han, J. H. (2014). Memory recall and modifications by activating neurons with elevated creb. Nat. Neurosci. 17, 65-72. doi: 10.1038/nn.3592

Kim, S. M., Ganguli, S., and Frank, L. M. (2012). Spatial information outflow from the hippocampal circuit: distributed spatial coding and phase precession in the subiculum. J. Neurosci. 32, 11539-11558. doi: 10.1523/JNEUROSCI.5942-11.2012

Klausberger, T., and Somogyi, P. (2008). Neuronal diversity and temporal dynamics: the unity of hippocampal circuit operations. Science 321, 53-57. doi: $10.1126 /$ science. 1149381

Kocsis, B., Bragin, A., and Buzsáki, G. (1999). Interdependence of multiple theta generators in the hippocampus: a partial coherence analysis. J. Neurosci. 19, 6200-6212. doi: 10.1523/JNEUROSCI.19-14-06200.1999

Konopacki, J., MacIver, M. B., Bland, B. H., and Roth, S. H. (1987). Carbacholinduced eeg 'theta' activity in hippocampal brain slices. Brain Res. 405, 196-198. doi: 10.1016/0006-8993(87)91009-2

Kramis, R., Vanderwolf, C. H., and Bland, B. H. (1975). Two types of hippocampal rhythmical slow activity in both the rabbit and the rat: relations to behavior and effects of atropine, diethyl ether, urethane, and pentobarbital. Exp. Neurol. 49(Pt 1):58-85. doi: 10.1016/0014-4886(75) 90195-8
Krupic, J., Burgess, N., and O'Keefe, J. (2012). Neural representations of location composed of spatially periodic bands. Science 337, 853-857. doi: $10.1126 /$ science. 1222403

Krupic, J., Burgess, N., and O'Keefe, J. (2015). Spatially periodic cells are neither formed from grids nor poor isolation. arXiv.org.

Lee, A. K., and Wilson, M. A. (2002). Memory of sequential experience in the hippocampus during slow wave sleep. Neuron 36, 1183-1194. doi: 10.1016/S0896-6273(02)01096-6

Lenck-Santini, P.-P., Fenton, A. A., and Muller, R. U. (2008). Discharge properties of hippocampal neurons during performance of a jump avoidance task. J. Neurosci. 28, 6773-6786. doi: 10.1523/JNEUROSCI.532907.2008

Lenck-Santini, P.-P., and Holmes, G. L. (2008). Altered phase precession and compression of temporal sequences by place cells in epileptic rats. J. Neurosci. 28, 5053-5062. doi: 10.1523/JNEUROSCI.5024-07.2008

Lengyel, M., Szatmáry, Z., and Erdi, P. (2003). Dynamically detuned oscillations account for the coupled rate and temporal code of place cell firing. Hippocampus 13, 700-714. doi: 10.1002/hipo.10116

Leutgeb, S., Leutgeb, J. K., Treves, A., Moser, M. B., and Moser, E. I. (2004). Distinct ensemble codes in hippocampal areas ca3 and cal. Science 305, 1295-1298. doi: $10.1126 /$ science. 1100265

Li, S., Cullen, W. K., Anwyl, R., and Rowan, M. J. (2003). Dopamine-dependent facilitation of ltp induction in hippocampal cal by exposure to spatial novelty. Nat. Neurosci. 6, 526-531. doi: 10.1038/nn1049

Lisman, J., and Buzsáki, G. (2008). A neural coding scheme formed by the combined function of gamma and theta oscillations. Schizophr. Bull. 34, 974980. doi: 10.1093/schbul/sbn060

Lisman, J., and Redish, A. D. (2009). Prediction, sequences and the hippocampus. Philos. Trans. R. Soc. Lond. B Biol. Sci. 364, 1193-1201. doi: $10.1098 /$ rstb.2008.0316

Lisman, J. E., and Idiart, M. A. (1995). Storage of $7+/-2$ short-term memories in oscillatory subcycles. Science $267,1512-1515$. doi: $10.1126 /$ science. 7878473

Lisman, J. E., and Jensen, O. (2013). The $\theta-\gamma$ neural code. Neuron 77, 1002-1016. doi: 10.1016/j.neuron.2013.03.007

Liu, X., Ramirez, S., Pang, P. T., Puryear, C. B., Govindarajan, A., Deisseroth, K., et al. (2012). Optogenetic stimulation of a hippocampal engram activates fear memory recall. Nature 484, 381-385. doi: 10.1038/nature11028

Losonczy, A., Zemelman, B. V., Vaziri, A., and Magee, J. C. (2010). Network mechanisms of theta related neuronal activity in hippocampal cal pyramidal neurons. Nat. Neurosci. 13, 967-972. doi: 10.1038/nn.2597

Lu, X., and Bilkey, D. K. (2010). The velocity-related firing property of hippocampal place cells is dependent on self-movement. Hippocampus 20, $573-583$.

Lubenov, E. V., and Siapas, A. G. (2009). Hippocampal theta oscillations are travelling waves. Nature 459, 534-539. doi: 10.1038/nature08010

Ma, S., Allocca, G., Ong-Plsson, E. K. E., Singleton, C. E., Hawkes, D., McDougall, S. J., et al. (2017). Nucleus incertus promotes cortical desynchronization and behavioral arousal. Brain Struct. Funct. 222, 515-537. doi: 10.1007/s00429-016-1230-0

Macdonald, C. J., Lepage, K. Q., Eden, U. T., and Eichenbaum, H. (2011). Hippocampal "time cells" bridge the gap in memory for discontiguous events. Neuron 71, 737-749. doi: 10.1016/j.neuron.2011.07.012

Magee, J. C. (2001). Dendritic mechanisms of phase precession in hippocampal cal pyramidal neurons. J. Neurophysiol. 86, 528-532. doi: 10.1152/jn.2001.86.1.528

Magee, J. C., and Johnston, D. (1997). A synaptically controlled, associative signal for hebbian plasticity in hippocampal neurons. Science 275, 209-213. doi: 10.1126/science.275.5297.209

Maingret, N., Girardeau, G., Todorova, R., Goutierre, M., and Zugaro, M. (2016). Hippocampo-cortical coupling mediates memory consolidation during sleep. Nat. Neurosci. 19, 959-964. doi: 10.1038/nn.4304

Malhotra, S., Cross, R. W. A., and van der Meer, M. A. A. (2012). Theta phase precession beyond the hippocampus. Rev. Neurosci. 23, 39-65. doi: 10.1515/revneuro-2011-0064

Markram, H., Lübke, J., Frotscher, M., and Sakmann, B. (1997). Regulation of synaptic efficacy by coincidence of postsynaptic aps and epsps. Science 275, 213-215. doi: 10.1126/science.275.5297.213

Marrosu, F., Portas, C., Mascia, M. S., Casu, M. A., Fà, M., Giagheddu, M., et al. (1995). Microdialysis measurement of cortical and hippocampal acetylcholine 
release during sleep-wake cycle in freely moving cats. Brain Res. 671, 329-332. doi: 10.1016/0006-8993(94)01399-3

Mattar, M. G., and Daw, N. D. (2018). Prioritized memory access explains planning and hippocampal replay. Nat. Neurosci. 21, 1609-1617. doi: 10.1038/s41593-018-0232-Z

Maurer, A. P., Cowen, S. L., Burke, S. N., Barnes, C. A., and McNaughton, B. L. (2006). Organization of hippocampal cell assemblies based on theta phase precession. Hippocampus 16, 785-794. doi: 10.1002/hipo.20202

Maurer, A. P., Lester, A. W., Burke, S. N., Ferng, J. J., and Barnes, C. A. (2014). Back to the future: preserved hippocampal network activity during reverse ambulation. J. Neurosci. 34, 15022-15031. doi: 10.1523/JNEUROSCI.1129-14.2014

Maurer, A. P., and McNaughton, B. L. (2007). Network and intrinsic cellular mechanisms underlying theta phase precession of hippocampal neurons. Trends Neurosci. 30, 325-333. doi: 10.1016/j.tins.2007.05.002

Maurer, A. P., Vanrhoads, S. R., Sutherland, G. R., Lipa, P., and McNaughton, B. L. (2005). Self-motion and the origin of differential spatial scaling along the septo-temporal axis of the hippocampus. Hippocampus 15, 841-852. doi: 10.1002/hipo. 20114

McFarland, W. L., Teitelbaum, H., and Hedges, E. K. (1975). Relationship between hippocampal theta activity and running speed in the rat. J. Compar. Physiol. Psychol. 88, 324-328. doi: 10.1037/h0076177

McNamara, C. G., Tejero-Cantero, A., Trouche, S., Campo-Urriza, N., and Dupret, D. (2014). Dopaminergic neurons promote hippocampal reactivation and spatial memory persistence. Nat. Neurosci. 17, 1658-1660. doi: 10.1038/nn.3843

McNaughton, B. L., Barnes, C. A., and O’Keefe, J. (1983). The contributions of position, direction, and velocity to single unit activity in the hippocampus of freely-moving rats. Exp. Brain Res. 52, 41-49. doi: 10.1007/BF00237147

Mehta, M. R., Barnes, C. A., and McNaughton, B. L. (1997). Experience-dependent, asymmetric expansion of hippocampal place fields. Proc. Natl. Acad. Sci. U.S.A. 94, 8918-8921. doi: 10.1073/pnas.94.16.8918

Mehta, M. R., Lee, A. K., and Wilson, M. A. (2002). Role of experience and oscillations in transforming a rate code into a temporal code. Nature 417, 741-746. doi: 10.1038/nature00807

Mehta, M. R., Quirk, M. C., and Wilson, M. A. (2000). Experience-dependent asymmetric shape of hippocampal receptive fields. Neuron 25, 707-715. doi: 10.1016/S0896-6273(00)81072-7

Middleton, S. J., and McHugh, T. J. (2016). Silencing ca3 disrupts temporal coding in the cal ensemble. Nat. Neurosci. 19, 945-951. doi: 10.1038/nn.4311

Miller, G. A. (1956). Magical number seven plus or minus two: some limits on our capacity for processing information. Psychol. Rev. 63, 81-97. doi: $10.1037 /$ h0043158

Mizuseki, K., Sirota, A., Pastalkova, E., and Buzsáki, G. (2009). Theta oscillations provide temporal windows for local circuit computation in the entorhinalhippocampal loop. Neuron 64, 267-280. doi: 10.1016/j.neuron.2009.08.037

Moita, M. A. P., Rosis, S., Zhou, Y., LeDoux, J. E., and Blair, H. T. (2003). Hippocampal place cells acquire location-specific responses to the conditioned stimulus during auditory fear conditioning. Neuron 37, 485-497. doi: 10.1016/S0896-6273(03)00033-3

Morris, R. G., Garrud, P., Rawlins, J. N., and O'Keefe, J. (1982). Place navigation impaired in rats with hippocampal lesions. Nature 297, 681-683. doi: $10.1038 / 297681 \mathrm{a} 0$

Muenzinger, K. F., and Gentry E. (1931). Tone discrimination in white rats. J. Comp. Psychol. 12, 195-206. doi: 10.1037/h0072238

Muessig, L., Lasek, M., Varsavsky, I., Cacucci, F., and Wills, T. J. (2019). Coordinated emergence of hippocampal replay and theta sequences during post-natal development. Curr. Biol. 29, 834-840.e4. doi: 10.1016/j.cub.2019.01.005

Müller, C., and Remy, S. (2014). Dendritic inhibition mediated by o-lm and bistratified interneurons in the hippocampus. Front. Synaptic Neurosci. 6:23. doi: $10.3389 /$ fnsyn.2014.00023

Navratilova, Z., Godfrey, K. B., and McNaughton, B. L. (2016). Grids from bands, or bands from grids? An examination of the effects of single unit contamination on grid cell firing fields. J. Neurophysiol. 115, 992-1002. doi: 10.1152/jn.00699.2015

O'Keefe, J. (1976). Place units in the hippocampus of the freely moving rat. Exp. Neurol. 51, 78-109. doi: 10.1016/0014-4886(76)90055-8
O'Keefe, J., and Dostrovsky, J. (1971). The hippocampus as a spatial map. preliminary evidence from unit activity in the freely-moving rat. Brain Res. 34, 171-175. doi: 10.1016/0006-8993(71)90358-1

O'Keefe, J., and Nadel, L. (1978). The Hippocampus as a Cognitive Map. Oxford: Clarendon Press.

O'Keefe, J., and Recce, M. L. (1993). Phase relationship between hippocampal place units and the EEG theta rhythm. Hippocampus 3, 317-330. doi: 10.1002/hipo.450030307

Ólafsdóttir, H. F., Carpenter, F., and Barry, C. (2016). Coordinated grid and place cell replay during rest. Nat. Neurosci. 19, 792-794. doi: 10.1038/nn.4291

Ólafsdóttir, H. F., Carpenter, F., and Barry, C. (2017). Task demands predict a dynamic switch in the content of awake hippocampal replay. Neuron 96, 925-935-e6. doi: 10.1101/172098

O’Neill, J., Boccara, C. N., Stella, F., Schoenenberger, P., and Csicsvari, J. (2017). Superficial layers of the medial entorhinal cortex replay independently of the hippocampus. Science 355, 184-188. doi: 10.1126/science.aag2787

O’Neill, J., Pleydell-Bouverie, B., Dupret, D., and Csicsvari, J. (2010). Play it again: reactivation of waking experience and memory. Trends Neurosci. 33, 220-229. doi: 10.1016/j.tins.2010.01.006

O'Neill, J., Senior, T., and Csicsvari, J. (2006). Place-selective firing of cal pyramidal cells during sharp wave/ripple network patterns in exploratory behavior. Neuron 49, 143-155. doi: 10.1016/j.neuron.2005.10.037

O'Neill, J., Senior, T. J., Allen, K., Huxter, J. R., and Csicsvari, J. (2008). Reactivation of experience-dependent cell assembly patterns in the hippocampus. Nat. Neurosci. 11, 209-215. doi: 10.1038/nn2037

Ormond, J., and McNaughton, B. L. (2015). Place field expansion after focal mec inactivations is consistent with loss of fourier components and path integrator gain reduction. Proc. Natl. Acad. Sci. U.S.A. 112, 4116-4121. doi: $10.1073 /$ pnas. 1421963112

Orzeł-Gryglewska, J., Matulewicz, P., and Jurkowlaniec, E. (2015). Brainstem system of hippocampal theta induction: the role of the ventral tegmental area. Synapse 69, 553-575. doi: 10.1002/syn.21843

Pan, W. X., and McNaughton, N. (1997). Medial supramammillary nucleus, spatial learning and the frequency of hippocampal theta activity. Brain Res. 764, 101-108. doi: 10.1016/S0006-8993(97)00431-9

Papale, A. E., Zielinski, M. C., Frank, L. M., Jadhav, S. P., and Redish, A. D. (2016). Interplay between hippocampal sharp-wave-ripple events and vicarious trial and error behaviors in decision making. Neuron 92, 975-982. doi: 10.1016/j.neuron.2016.10.028

Pastalkova, E., Itskov, V., Amarasingham, A., and Buzsáki, G. (2008). Internally generated cell assembly sequences in the rat hippocampus. Science 321, 13221327. doi: 10.1126/science. 1159775

Patel, J., Fujisawa, S., Berényi, A., Royer, S., and Buzsáki, G. (2012). Traveling theta waves along the entire septotemporal axis of the hippocampus. Neuron 75, 410-417. doi: 10.1016/j.neuron.2012.07.015

Pavlides, C., and Winson, J. (1989). Influences of hippocampal place cell firing in the awake state on the activity of these cells during subsequent sleep episodes. J. Neurosci. 9, 2907-2918. doi: 10.1523/JNEUROSCI.09-08-02907.1989

Peck, B. K., and Vanderwolf, C. H. (1991). Effects of raphe stimulation on hippocampal and neocortical activity and behaviour. Brain Res. 568, 244-252. doi: 10.1016/0006-8993(91)91404-O

Petersen, R. S., Panzeri, S., and Diamond, M. E. (2001). Population coding of stimulus location in rat somatosensory cortex. Neuron 32, 503-514. doi: 10.1016/S0896-6273(01)00481-0

Petsche, H., Stumpf, C., and Gogolak, G. (1962). [the significance of the rabbit's septum as a relay station between the midbrain and the hippocampus. I. The control of hippocampus arousal activity by the septum cells]. Electroencephalogr. Clin. Neurophysiol. 14, 202-211. doi: 10.1016/0013-4694(62)90030-5

Pfeiffer, B. E., and Foster, D. J. (2013). Hippocampal place-cell sequences depict future paths to remembered goals. Nature 497, 74-79. doi: 10.1038/nature12112

Poo, M. M., Pignatelli, M., Ryan, T. J., Tonegawa, S., Bonhoeffer, T., Martin, K. C., et al. (2016). What is memory? The present state of the engram. BMC Biol. 14:40. doi: 10.1186/s12915-016-0261-6

Qasim, S. E., and Jacobs, J. (2016). Human hippocampal theta oscillations during movement without visual cues. Neuron 89, 1121-1123. doi: 10.1016/j.neuron.2016.03.003 
Raudies, F., Brandon, M. P., Chapman, G. W., and Hasselmo, M. E. (2015). Head direction is coded more strongly than movement direction in a population of entorhinal neurons. Brain Res. 1621, 355-367. doi: 10.1016/j.brainres.2014.10.053

Ravassard, P., Kees, A., Willers, B., Ho, D., Aharoni, D., Cushman, J., et al. (2013). Multisensory control of hippocampal spatiotemporal selectivity. Science 340, 1342-1346. doi: 10.1126/science.1232655

Redish, A. D. (2016). Vicarious trial and error. Nat. Rev. Neurosci. 17, 147-159. doi: $10.1038 / \mathrm{nrn} .2015 .30$

Redondo, R. L., Kim, J., Arons, A. L., Ramirez, S., Liu, X., and Tonegawa, S. (2014). Bidirectional switch of the valence associated with a hippocampal contextual memory engram. Nature 513, 426-430. doi: 10.1038/nature13725

Reifenstein, E., Stemmler, M., Herz, A. V. M., Kempter, R., and Schreiber, S. (2014). Movement dependence and layer specificity of entorhinal phase precession in two-dimensional environments. PLOS ONE 9:e100638. doi: 10.1371/journal.pone.0100638

Reifenstein, E. T., Ebbesen, C. L., Tang, Q., Brecht, M., Schreiber, S., and Kempter, R. (2016). Cell-type specific phase precession in layer ii of the medial entorhinal cortex. J. Neurosci. 36, 2283-2288. doi: 10.1523/JNEUROSCI.2986-15.2016

Reifenstein, E. T., Kempter, R., Schreiber, S., Stemmler, M. B., and Herz, A. V. M. (2012). Grid cells in rat entorhinal cortex encode physical space with independent firing fields and phase precession at the single-trial level. Proc. Natl. Acad. Sci. U.S.A. 109, 6301-6306. doi: 10.1073/pnas.1109599109

Reijmers, L. G., Perkins, B. L., Matsuo, N., and Mayford, M. (2007). Localization of a stable neural correlate of associative memory. Science 317, 1230-1233. doi: 10.1126/science.1143839

Riehle, A., Grün, S., Diesmann, M., and Aertsen, A. (1997). Spike synchronization and rate modulation differentially involved in motor cortical function. Science 278, 1950-1953. doi: 10.1126/science.278.5345.1950

Robbe, D., and Buzsáki, G. (2009). Alteration of theta timescale dynamics of hippocampal place cells by a cannabinoid is associated with memory impairment. J. Neurosci. 29, 12597-12605. doi: 10.1523/JNEUROSCI.2407-09.2009

Robbe, D., Montgomery, S. M., Thome, A., Rueda-Orozco, P. E., McNaughton, B. L., and Buzsaki, G. (2006). Cannabinoids reveal importance of spike timing coordination in hippocampal function. Nat. Neurosci. 9, 1526-1533. doi: $10.1038 / \mathrm{nn} 1801$

Robinson, N. T. M., Priestley, J. B., Rueckemann, J. W., Garcia, A. D., Smeglin, V. A., Marino, F. A., et al. (2017). Medial entorhinal cortex selectively supports temporal coding by hippocampal neurons. Neuron 94, 677-688-e6. doi: 10.1016/j.neuron.2017.04.003

Romani, S., and Tsodyks, M. (2015). Short-term plasticity based network model of place cells dynamics. Hippocampus 25, 94-105. doi: 10.1002/hipo.22355

Roumis, D. K., and Frank, L. M. (2015). Hippocampal sharp-wave ripples in waking and sleeping states. Curr. Opin. Neurobiol. 35, 6-12. doi: 10.1016/j.conb.2015.05.001

Roux, L., Hu, B., Eichler, R., Stark, E., and Buzsáki, G. (2017). Sharp wave ripples during learning stabilize the hippocampal spatial map. Nat. Neurosci. 20, 845-853. doi: 10.1038/nn.4543

Royer, S., Zemelman, B. V., Losonczy, A., Kim, J., Chance, F., Magee, J. C., et al. (2012). Control of timing, rate and bursts of hippocampal place cells by dendritic and somatic inhibition. Nat. Neurosci. 15, 769-775. doi: $10.1038 / \mathrm{nn} .3077$

Sadowski, J. H. L. P., Jones, M. W., and Mellor, J. R. (2016). Sharp-wave ripples orchestrate the induction of synaptic plasticity during reactivation of place cell firing patterns in the hippocampus. Cell Rep. 14, 1916-1929. doi: 10.1016/j.celrep.2016.01.061

Saravanan, V., Arabali, D., Jochems, A., Cui, A. X., Gootjes-Dreesbach, L., Cutsuridis, V., et al. (2015). Transition between encoding and consolidation/replay dynamics via cholinergic modulation of can current: a modeling study. Hippocampus 25, 1052-1070. doi: 10.1002/hipo.22429

Schlesiger, M. I., Cannova, C. C., Boublil, B. L., Hales, J. B., Mankin, E. A., Brandon, M. P., et al. (2015). The medial entorhinal cortex is necessary for temporal organization of hippocampal neuronal activity. Nat. Neurosci. 18, 1123-1132. doi: $10.1038 / \mathrm{nn} .4056$

Schmidt, B., Papale, A., Redish, A. D., and Markus, E. J. (2013). Conflict between place and response navigation strategies: effects on vicarious trial and error (vte) behaviors. Learn. Mem. 20, 130-138. doi: 10.1101/lm.028753.112
Schmidt, R., Diba, K., Leibold, C., Schmitz, D., Buzsáki, G., and Kempter, R. (2009) Single-trial phase precession in the hippocampus. J. Neurosci. 29, 13232-13241. doi: 10.1523/JNEUROSCI.2270-09.2009

Schmidt-Hieber, C. and Häusser, M. (2013). Cellular mechanisms of spatial navigation in the medial entorhinal cortex. Nat. Neurosci. 16, 325-331. doi: $10.1038 / \mathrm{nn} .3340$

Schomburg, E. W., Fernández-Ruiz, A., Mizuseki, K., Berényi, A., Anastassiou, C. A., Koch, C., et al. (2014). Theta phase segregation of input-specific gamma patterns in entorhinal-hippocampal networks. Neuron 84, 470-485. doi: 10.1016/j.neuron.2014.08.051

Scoville, W. B., and Milner, B. (1957). Loss of recent memory after bilateral hippocampal lesions. J. Neurol. Neurosurg. Psychiatry 20, 11-21. doi: 10.1136/jnnp.20.1.11

Semon, R. (1921). The Mneme. London: George Allen \& Unwin Ltd Edition.

Senior, T. J., Huxter, J. R., Allen, K., O’Neill, J., and Csicsvari, J. (2008). Gamma oscillatory firing reveals distinct populations of pyramidal cells in the cal region of the hippocampus. J. Neurosci. 28, 2274-2286. doi: 10.1523/JNEUROSCI.4669-07.2008

Sheremet, A., Burke, S. N., and Maurer, A. P. (2016). Movement enhances the nonlinearity of hippocampal theta. J. Neurosci. 36, 4218-4230. doi: 10.1523/JNEUROSCI.3564-15.2016

Silva, A. J., Zhou, Y., Rogerson, T., Shobe, J., and Balaji, J. (2009). Molecular and cellular approaches to memory allocation in neural circuits. Science 326, 391-395. doi: 10.1126/science.1174519

Silva, D., Feng, T., and Foster, D. J. (2015). Trajectory events across hippocampal place cells require previous experience. Nat. Neurosci. 18, 1772-1779. doi: 10.1038/nn.4151

Singer, A. C., Carr, M. F., Karlsson, M. P., and Frank, L. M. (2013). Hippocampal SWR activity predicts correct decisions during the initial learning of an alternation task. Neuron 77, 1163-1173. doi: 10.1016/j.neuron.2013. 01.027

Singer, A. C., and Frank, L. M. (2009). Rewarded outcomes enhance reactivation of experience in the hippocampus. Neuron 64, 910-921. doi: 10.1016/j.neuron.2009.11.016

Sinnamon, H. M. (1993). Preoptic and hypothalamic neurons and the initiation of locomotion in the anesthetized rat. Prog. Neurobiol. 41, 323-344. doi: 10.1016/0301-0082(93)90003-B

Skaggs, W. E., McNaughton, B. L., Wilson, M. A., and Barnes, C. A. (1996). Theta phase precession in hippocampal neuronal populations and the compression of temporal sequences. Hippocampus 6, 149-172.

Sńska, U., and Kasicki, S. (1998). The frequency of rat's hippocampal theta rhythm is related to the speed of locomotion. Brain Res. 796, 327-331. doi: 10.1016/S0006-8993(98)00390-4

Song, E. Y., Kim, Y. B., Kim, Y. H., and Jung, M. W. (2005a). Role of active movement in place-specific firing of hippocampal neurons. Hippocampus 15 8-17. doi: 10.1002/hipo.20023

Song, S., Sjöström, P. J., Reigl, M., Nelson, S., and Chklovskii, D. B. (2005b). Highly nonrandom features of synaptic connectivity in local cortical circuits. PLoS Biol. 3:e68. doi: 10.1371/journal.pbio.0030068

Stopfer, M., Bhagavan, S., Smith, B. H., and Laurent, G. (1997). Impaired odour discrimination on desynchronization of odour-encoding neural assemblies. Nature 390, 70-74. doi: 10.1038/36335

Stuart, G. J. (2001). Determinants of spike timing-dependent synaptic plasticity. Neuron 32, 966-968. doi: 10.1016/S0896-6273(01)00541-4

Takahashi, M., Nishida, H., Redish, A. D., and Lauwereyns, J. (2014). Theta phase shift in spike timing and modulation of gamma oscillation: a dynamic code for spatial alternation during fixation in rat hippocampal area cal. J. Neurophysiol. 111, 1601-1614. doi: 10.1152/jn.00395.2013

Takahashi, S. (2015). Episodic-like memory trace in awake replay of hippocampal place cell activity sequences. Elife 4:e08105. doi: 10.7554/eLife.08105

Takahashi, Y. K., Langdon, A. J., Niv, Y., and Schoenbaum, G. (2016). Temporal specificity of reward prediction errors signaled by putative dopamine neurons in rat vta depends on ventral striatum. Neuron 91, 182-193. doi: 10.1016/j.neuron.2016.05.015

Takeuchi, T., Duszkiewicz, A. J., Sonneborn, A., Spooner, P. A., Yamasaki, M., Watanabe, M., et al. (2016). Locus coeruleus and dopaminergic consolidation of everyday memory. Nature 537, 357-362. doi: 10.1038/nature 19325 
Terada, S., Sakurai, Y., Nakahara, H., and Fujisawa, S. (2017). Temporal and rate coding for discrete event sequences in the hippocampus. Neuron 94, 1248-1262-e4. doi: 10.1016/j.neuron.2017.05.024

Terrazas, A., Krause, M., Lipa, P., Gothard, K. M., Barnes, C. A., and McNaughton, B. L. (2005). Self-motion and the hippocampal spatial metric. J. Neurosci. 25, 8085-8096. doi: 10.1523/JNEUROSCI.0693-05.2005

Tolman, E. C. (1948). Cognitive Maps in Rats and Men, Vol. 55.

Tonegawa, S., Pignatelli, M., Roy, D. S., and Ryan, T. J. (2015). Memory engram storage and retrieval. Curr. Opin. Neurobiol. 35, 101-109. doi: 10.1016/j.conb.2015.07.009

Tóth, K., Borhegyi, Z., and Freund, T. F. (1993). Postsynaptic targets of gabaergic hippocampal neurons in the medial septum-diagonal band of broca complex. J. Neurosci. 13, 3712-3724. doi: 10.1523/JNEUROSCI.13-09-03712.1993

Trouche, S., Perestenko, P. V., van de Ven, G. M., Bratley, C. T., McNamara, C. G., Campo-Urriza, N., et al. (2016). Recoding a cocaine-place memory engram to a neutral engram in the hippocampus. Nat. Neurosci. 19, 564-567. doi: $10.1038 / \mathrm{nn} .4250$

Tsao, A., Moser, M. B., and Moser, E. I. (2013). Traces of experience in the lateral entorhinal cortex. Curr. Biol. 23, 399-405. doi: 10.1016/j.cub.2013.01.036

Tsodyks, M. V., Skaggs, W. E., Sejnowski, T. J., and McNaughton, B. L. (1996). Population dynamics and theta rhythm phase precession of hippocampal place cell firing: a spiking neuron model. Hippocampus 6, 271-280.

van de Ven, G. M., Trouche, S., McNamara, C. G., Allen, K., and Dupret, D. (2016). Hippocampal offline reactivation consolidates recently formed cell assembly patterns during sharp wave-ripples. Neuron 92, 968-974. doi: 10.1016/j.neuron.2016.10.020

van der Meer, M. A. A., and Redish, A. D. (2011). Theta phase precession in rat ventral striatum links place and reward information. J. Neurosci. 31, 2843-2854. doi: 10.1523/JNEUROSCI.4869-10.2011

van Strien, N. M., Cappaert, N. L. M., and Witter, M. P. (2009). The anatomy of memory: an interactive overview of the parahippocampal-hippocampal network. Nat. Rev. Neurosci. 10, 272-282. doi: 10.1038/nrn2614

Vanderwolf, C. H. (1969). Hippocampal electrical activity and voluntary movement in the rat. Electroencephalogr. Clin. Neurophysiol. 26, 407-418. doi: 10.1016/0013-4694(69)90092-3

Vanderwolf, C. H., Buzsaki, G., Cain, D. P., Cooley, R. K., and Robertson, B. (1988). Neocortical and hippocampal electrical activity following decapitation in the rat. Brain Res. 451, 340-344. doi: 10.1016/0006-8993(88) 90780-9

Vertes, R. P., Hoover, W. B., and Prisco, G. V. D. (2004). Theta rhythm of the hippocampus: subcortical control and functional significance. Behav. Cogn. Neurosci. Rev. 3, 173-200. doi: 10.1177/1534582304273594

Vertes, R. P., and Kocsis, B. (1997). Brainstem-diencephalo-septohippocampal systems controlling the theta rhythm of the hippocampus. Neuroscience 81, 893-926.

Wang, D. V., Yau, H. J., Broker, C. J., Tsou, J. H., Bonci, A., and Ikemoto, S. (2015). Mesopontine median raphe regulates hippocampal ripple oscillation and memory consolidation. Nat. Neurosci. 18, 728-735. doi: 10.1038/nn.3998

Wang, Y., Roth, Z., and Pastakova, E. (2016). Synchronized excitability in a network enables generation of internal neuronal sequences. Elife 5:e20697. doi: 10.7554/eLife.20697

Welday, A. C., Shlifer, I. G., Bloom, M. L., Zhang, K., and Blair, H. T. (2011). Cosine directional tuning of theta cell burst frequencies: evidence for spatial coding by oscillatory interference. J. Neurosci. 31, 16157-16176. doi: 10.1523/JNEUROSCI.0712-11.2011

Wikenheiser, A. M., and Redish, A. D. (2013). The balance of forward and backward hippocampal sequences shifts across behavioral states. Hippocampus 23, 22-29. doi: 10.1002/hipo.22049

Wikenheiser, A. M., and Redish, A. D. (2015a). Decoding the cognitive map: ensemble hippocampal sequences and decision making. Curr. Opin. Neurobiol. 32, 8-15. doi: 10.1016/j.conb.2014.10.002

Wikenheiser, A. M., and Redish, A. D. (2015b). Hippocampal theta sequences reflect current goals. Nat. Neurosci. 18, 289-294. doi: 10.1038/nn.3909

Wilson, M. A., and McNaughton, B. L. (1994). Reactivation of hippocampal ensemble memories during sleep. Science 265, 676-679. doi: 10.1126/science.8036517

Witter, M. P., Groenewegen, H. J., da Silva, F. H. L., and Lohman, A. H. (1989). Functional organization of the extrinsic and intrinsic circuitry of the parahippocampal region. Prog. Neurobiol. 33, 161-253. doi: 10.1016/0301-0082(89)90009-9

Wójtowicz, T. and Mozrzymas, J. W. (2015). Diverse impact of neuronal activity at $\theta$ frequency on hippocampal long-term plasticity. J. Neurosci. Res. 93, 1330-1344. doi: 10.1002/jnr.23581

Wood, E. R., Dudchenko, P. A., and Eichenbaum, H. (1999). The global record of memory in hippocampal neuronal activity. Nature 397, 613-616. doi: $10.1038 / 17605$

Wu, C. T., Haggerty, D., Kemere, C., and Ji, D. (2017). Hippocampal awake replay in fear memory retrieval. Nat. Neurosci. 20, 571-580. doi: 10.1038/nn.4507

Yamaguchi, Y. (2003). A theory of hippocampal memory based on theta phase precession. Biol. Cybern. 89, 1-9.

Yu, J. Y., and Frank, L. M. (2015). Hippocampal-cortical interaction in decision making. Neurobiol. Learn. Mem. 117, 34-41. doi: 10.1016/j.nlm.2014.02.002

Zheng, C., Bieri, K. W., Hsiao, Y. T., and Colgin, L. L. (2016). Spatial sequence coding differs during slow and fast gamma rhythms in the hippocampus. Neuron 89, 398-408. doi: 10.1016/j.neuron.2015.12.005

Zielinski, M. C., Tang, W., and Jadhav, S. P. (2017). The role of replay and theta sequences in mediating hippocampal-prefrontal interactions for memory and cognition. Hippocampus. doi: 10.1002/hipo.22821. [Epub ahead of print].

Zugaro, M. B., Monconduit, L., and Buzsáki, G. (2005). Spike phase precession persists after transient intrahippocampal perturbation. Nat. Neurosci. 8, 67-71. doi: $10.1038 / \mathrm{nn} 1369$

Zutshi, I., Brandon, M. P., Fu, M. L., Donegan, M. L., Leutgeb, J. K., and Leutgeb, S. (2018). Hippocampal neural circuits respond to optogenetic pacing of theta frequencies by generating accelerated oscillation frequencies. Curr. Biol. 28, 1179-1188-e3. doi: 10.1016/j.cub.2018.02.061

Conflict of Interest Statement: The authors declare that the research was conducted in the absence of any commercial or financial relationships that could be construed as a potential conflict of interest.

Copyright (C) 2019 Drieu and Zugaro. This is an open-access article distributed under the terms of the Creative Commons Attribution License (CC BY). The use, distribution or reproduction in other forums is permitted, provided the original author(s) and the copyright owner(s) are credited and that the original publication in this journal is cited, in accordance with accepted academic practice. No use, distribution or reproduction is permitted which does not comply with these terms. 


\section{GLOSSARY}

Cell assembly. Group of neurons that transiently co-activate during a certain function or mental process. Place cells with highly similar fields can fire within a brief window of time (common theta phase, often grouped within a gamma cycle) and form a cell assembly. Reciprocal connectivity between group members may or may not be implied (e.g., cell assemblies in CA3 vs. CA1).

Theta sequence. Sequential activation of hippocampal cell assemblies paced by the ongoing theta rhythm $(\sim 8 \mathrm{~Hz})$ and representing the ongoing trajectory at a highly accelerated rate. For instance, when a rat successively traverses place fields A, B, and C (which lasts on the order of a second), in the overlapping region, ongoing theta cycles contain spikes $\mathrm{A}, \mathrm{B}$, and $\mathrm{C}$ in the same order (within only $\sim 150 \mathrm{~ms}$ ). Theta sequences typically span one theta cycle, but longer sequences have also been described.

Replay. Reinstatement during offline states of the sequential activity observed during behavior. Replay is associated with sharp wave ripple complexes and can occur both in the forward or backward direction.

Theta phase precession. The time at which a cell fires relative to the ongoing theta cycle ("phase") decreases as the animal moves through space. Thus, the cell initially fires at the end of a theta cycle, then slightly before the end of the next cycle, until it eventually fires its last spikes near the beginning of the ongoing theta cycle. This phenomenon is called "phase precession." Initially described in place cells, phase precession has also been reported in other hippocampal cells, including interneurons, "episode," and sound-responsive cells (in which case the movement is considered in a more abstract space), as well as in other brain structures such as the medial prefrontal cortex or the ventral striatum.

Nested oscillations. Intermingled oscillations of different frequencies, especially when the phase of the slower oscillation modulates the amplitude of the faster oscillation. For instance, slow, medium and fast gamma bursts tend to occur at different phases of theta (phase-amplitude modulation).

Nested sequences. As a rat explores an environment, place cell assemblies activate in sequence and thus form "behavioral" (slow time scale) sequences representing the ongoing trajectory in real time. Simultaneously, the same assemblies form theta (fast time scale) sequences that describe the same trajectory, nested within the behavioral sequences. 